\title{
Anterior Cingulate Cortex to Ventral Hippocampus Circuit Mediates Contextual Fear Generalization
}

\author{
Xin-Lan Bian, ${ }^{1 \star}$ Cheng Qin, ${ }^{1 *}$ Cheng-Yun Cai, ${ }^{1 \star}$ Ying Zhou, ${ }^{1}$ Yan Tao, ${ }^{1}$ Yu-Hui Lin, ${ }^{1}$ Hai-Yin Wu, ${ }^{1,3}$ Lei Chang, ${ }^{1}$ \\ (DChun-Xia Luo, ${ }^{1,3}$ and Dong-Ya Zhu ${ }^{1,2,3}$ \\ ${ }^{1}$ Department of Pharmacology and ${ }^{2}$ Institution of Stem Cells and Neuroregeneration, Nanjing Medical University, Nanjing 211166, China, and ${ }^{3} \mathrm{Guangdong}-$ \\ Hong Kong-Macao Greater Bay Area Center for Brain Science and Brain-Inspired Intelligence, Guangzhou 510000, China
}

Contextual fear memory becomes less context-specific over time, a phenomenon referred to as contextual fear generalization. Overgeneralization of contextual fear memory is a core symptom of post-traumatic stress disorder (PTSD), but circuit mechanisms underlying the generalization remain unclear. We show here that neural projections from the anterior cingulate cortex (ACC) to ventral hippocampus (vHPC) mediate contextual fear generalization in male mice. Retrieval of contextual fear in a novel context at a remote time point activated cells in the ACC and vHPC, as indicated by significantly increased C-fos ${ }^{+}$cells. Using chemogenetic or photogenetic manipulations, we observed that silencing the activity of ACC or VHPC neurons reduced contextual fear generalization at the remote time point, whereas stimulating the activity of ACC or VHPC neurons facilitated contextual fear generalization at a recent time point. We found that ACC neurons projected to the vHPC unidirectionally, and importantly, silencing the activity of projection fibers from the ACC to vHPC inhibited contextual fear generalization at the remote time point. Together, our findings reveal an ACC to vHPC circuit that controls expression of fear generalization and may offer new strategies to prevent or reverse contextual fear generalization in subjects with anxiety disorders, especially in PTSD.

Key words: anterior cingulate cortex; anxiety disorders; contextual fear memory; memory generalization; neural circuit; ventral hippocampus

\section{Significance Statement}

Overgeneralization of contextual fear memory is a cardinal feature of PTSD, but circuit mechanisms underlying it remain unclear. Our study indicates that neural projections from the anterior cingulate cortex to ventral hippocampus control the expression of contextual fear generalization. Thus, manipulating the circuit may prevent or reverse fear overgeneralization in subjects with PTSD.

\section{Introduction}

Within a species, individual animals adopt various defensive behaviors to resist natural enemies and guard against risks in the environment. The defensive strategies are critical for the survival of an organism. Although defensive behaviors are often adaptive, pathologic expression of these defensive behaviors often leads to

Received 0ct. 23, 2018; revised April 29, 2019; accepted May 11, 2019.

Author contributions: D.-Y.Z. designed research; C.Q., X.-L.B., C.-Y.C., Y.Z., Y.T., and Y.-H.L. performed research; C.Q., X.-L.B., and Y.-H.L. analyzed data; H.-Y.W., L.C., and C.-X.L. contributed unpublished reagents/analytic tools; D.-Y.Z. wrote the paper.

This work was supported by Grants from the National Natural Science Foundation of China (31530091, 81870912), National Key Research and Development Program of China (2016YFC1306703), Science and Technology Program of Guangdong (2018B030334001), and by the Collaborative Innovation Center for Cardiovascular Disease Translational Medicine.

The authors declare no competing financial interests.

${ }^{*}$ X.-L.B., C.Q., and C.-Y.C. contributed equally to this work.

Correspondence should be addressed to Dong-Ya Zhu at dyzhu@njmu.edu.cn.

https://doi.org/10.1523/JNEUROSCI.2739-18.2019

Copyright $\odot 2019$ the authors inappropriate anxiety (Rosen and Schulkin, 1998; Bonne et al., 2004; McCullough et al., 2016). Indeed, anxiety-related disorders can be characterized by an inability to discriminate threat from safety (Dunsmoor et al., 2017). This is evident with posttraumatic stress disorder (PTSD), in which the re-experiencing of a past trauma is triggered by cues existing in a normally safe environment (Mahan and Ressler, 2012). The pathologic expression of defensive behaviors stems from that memory precision for recent events gives way to more gist-like representations over time, a phenomenon referred to as memory generalization (Jasnow et al., 2012; Wiltgen and Tanaka, 2013), which enables an animal to quickly and appropriately respond to novel stimuli that resemble a previous experience (Xu et al., 2013; Guo et al., 2018). To develop new treatments for clinical populations with anxiety disorders, an understanding of the fundamental circuits mediating contextual memory generalization is necessary.

In recent years, memory generalization has been extensively characterized, and multiple theories have been developed to ex- 
plain it. In addition to the hippocampus, part of the limbic system critical for maintaining the specificity of memories (Clelland et al., 2009; Fanselow and Dong, 2010; Ruediger et al., 2011; Alvares Lde et al., 2012), some researchers recently found that the medial prefrontal cortex (mPFC), thalamic nucleus reuniens (NR) and amygdala are essential for memory generalization (Antunes and Moita, 2010; Xu et al., 2013; Ashwell and Ito, 2014; Ghosh and Chattarji, 2015; Allsop et al., 2018; Grosso et al., 2018; Rozeske et al., 2018). According to memory consolidation hypotheses, the interaction between hippocampus and cortex promotes transformation of memory for long-term storage in the cortex. Over time, memories of previous experiences become less dependent on the hippocampus, and increasingly dependent on cortical regions, such as the ACC (Bontempi et al., 1999; Frankland et al., 2004; Goshen et al., 2011). Although the ACC can be considered a posterior portion of $\mathrm{mPFC}$, it is distinguished from typical $\mathrm{mPFC}$ at the level of the prelimbic cortex (Wang and Ikemoto, 2016). Inactivation of the ACC at remote time points reduces generalized fear expression and returns the memory to contextually precise form (Cullen et al., 2015; Einarsson et al., 2015), involving the ACC in contextual fear generalization. There are bidirectional communications between the dorsal hippocampus (dHPC) and ACC (Rajasethupathy et al., 2015). Precise context fear memory depends on the dHPC (Gafford et al., 2013). Different from the dHPC, which corresponds to the posterior hippocampus in primates and performs primarily cognitive functions, the vHPC (anterior in primates) relates to stress, emotion, and mood (Fanselow and Dong, 2010). Recently, a report showed that inactivation of vHPC by local lidocaine infusion attenuates fear generalization (Cullen et al., 2015), implicating the vHPC in the expression of fear generalization. However, whether there are neural projections between the VHPC and ACC, and if yes, whether the projections mediate contextual fear generalization remains unknown. Bridging this gap may identify new strategies to prevent or reverse contextual fear generalization in subjects with PTSD. Here, we identified a new neural circuit from the ACC to vHPC that controls contextual fear memory generalization.

\section{Materials and Methods}

Animals. Male young adult (6-8 weeks) C57BL/6 mice (from Mode Animal Research Center of Nanjing University, China) were used in this study. Animals were maintained at controlled temperature $\left(20 \pm 2^{\circ} \mathrm{C}\right)$ and group housed ( $12 \mathrm{~h}$ light/dark cycle) with access to food and water ad libitum. Every effort was made to minimize the number of animals used and their suffering. Microinfusions were performed while the mice were head-restrained on a stereotax. To adapt animals to the experimental environment, we handled animals for 3 min per day for 3 consecutive days before any procedure. All animal experiments were conducted in accordance with the Institutional Animal Care and Use Committee of Nanjing Medical University.

Recombinant viruses and their stereotaxic microinjections. Recombinant viruses adeno-associated virus 9 (AAV9)-hSyn-HA-hM4Di-IRESmCitrine (AAV00093), AAV9-CaMKII $\alpha$-ChR2-EYFP (AAV00027), and AAV9-CaMKII $\alpha$-EYFP (AAV00042) were purchased from Genechem. AAV9-CaMKII $\alpha$-hM3Dq-mCherry (PT-0049) and rAAV2/R-hSynEGFP-WPRE-pA (PT-0241) were purchased from BrainVTA. Mice were anesthetized with isoflurane and placed in a stereotaxic device. Solution of virus was microinjected with a glass pipette at a flow rate of 0.15 $\mu \mathrm{l} / \mathrm{min}$. The coordinates were AP: $+1.0 \mathrm{~mm}, \mathrm{ML}: \pm 0.3 \mathrm{~mm}, \mathrm{DV}$ : $-1.4 \mathrm{~mm}$ used for the ACC injection; AP: $+1.0 \mathrm{~mm}, \mathrm{ML}: \pm 0.5 \mathrm{~mm}$, DV: $-0.5 \mathrm{~mm}$ used for the M2 injection; AP: $-1.7 \mathrm{~mm}, \mathrm{ML}: \pm 1.0 \mathrm{~mm}$, DV: $-1.8 \mathrm{~mm}$ for the dHPC and AP: $-2.9 \mathrm{~mm}, \mathrm{ML}: \pm 2.8 \mathrm{~mm}, \mathrm{DV}:-3.6$ $\mathrm{mm}$ for the vHPC. The injection volume was $0.4 \mu \mathrm{l}$ per site in ACC, $0.3 \mu \mathrm{l}$ per site in M2, $0.6 \mu$ l per site in $\mathrm{AHPC}$ and vHPC. The microinjections were bilateral except otherwise noted.
$\mathrm{CNO}$ and its stereotaxic microinjection. Clozapine- $\mathrm{N}$-oxide (CNO; 4936) was purchased from Tocris Bioscience. CNO was dissolved with saline when in use. To investigate whether the neural projections from the ACC to vHPC mediate fear memory generalization, we implanted stainless-steel guide cannula (26 gauge, $3.5 \mathrm{~mm}$; Plastics One, RWD Life Science) into the vHPC ( $2.8 \mathrm{~mm}$ lateral from bregma, and $1.5 \mathrm{~mm}$ vertical from the cortical surface) immediately after the microinjection of viral vector into the vHPC. The cannula was fixed to the skull with adhesive luting cement and acrylic dental cement. Following surgery, a stainless-steel obturator was inserted into the guide cannula to avoid obstruction until microinjection was made. Mice were briefly headrestrained while the stainless-steel obturator was removed and a microinjection tube (30 gauge, $4.0 \mathrm{~mm}$, RWD Life Science) was inserted into the guide cannula. CNO (0.5 mm; Ye et al., 2017) was infused into the vHPC through the microinjection tube at a flow rate of $0.15 \mu \mathrm{l} / \mathrm{min}$ and the injection volume was $0.8 \mu \mathrm{l}$ per site.

Contextual fear conditioning and testing. Four to 6 weeks after virus microinjection, mice were habituated to handling for 3 consecutive days before contextual fear-conditioning training. The Training context ( $\mathrm{T}$ Ctx) consisted of the context chamber, with white noise $(60 \mathrm{~dB})$, dim illumination (house light), and stainless steel grid floors cleaned with $75 \%$ ethanol. The Novel context (N-Ctx) consisted of identical chambers, with a polka-dot insert attached to the rear wall, was illuminated only by yellow fluorescent lamp and contained no white noise. In addition, a flat, brown floor replaced the grid floor and was washed with $1 \%$ acetic acid. The contextual fear conditioning was performed with a computerized fear conditioning system (CSI Systems). On Day 1, mice were placed in T-Ctx $(30 \times 30 \mathrm{~cm}$ square arena $)$ for 3 min acclimation to record baseline behavior, and immediately after baseline recording, training was performed by delivering three foot shocks (lasting $2 \mathrm{~s}$ each at an intensity of $0.75 \mathrm{~mA}$, intertrial interval $60 \mathrm{~s}$ ) via a grid floor. T-Ctx was cleaned with $75 \%$ ethanol following all behavioral procedures. Retrieval of fear memory consisted of a 3 min exposure in either T-Ctx or N-Ctx and was performed on Day 2 (recent recall) or Day 15 (remote recall). The freezing level, defined as the absence of movements other than those required for breathing, was quantified from digitized video images using commercially available software (Freezescan, Clever Systems). To silence or stimulate the activity of ACC, dHPC, or vHPC neurons by chemogenetic manipulations, CNO was administered intraperitoneally ( $2 \mathrm{mg} / \mathrm{kg})$ $1 \mathrm{~h}$ before retrieval. To selectively silence the activity of projection fibers from the ACC to vHPC neurons, CNO was infused into the vHPC $30 \mathrm{~min}$ before retrieval. For the optogenetics experiment, mice were implanted with implantable fiber-optic light guides consisting of a $2.5 \mathrm{~mm}$ diameter metal ferrule with $0.22 \mathrm{NA}$ and a $200 \mathrm{~mm}$ thick protruding cleaved bare optic fiber cut to the $1.2 \mathrm{~mm}$ length (Thorlabs) in the ACC. During fear memory retrieval, optical stimulation through the fiberoptic connector was administered by delivering light through a patch cord connected to a $476 \mathrm{~nm}$ laser in $30 \mathrm{~s}$ light-on/30 s light-off sessions. During light-on sessions, stimulation was delivered at $20 \mathrm{~Hz}, 15 \mathrm{~ms}$ pulses, with $8-10 \mathrm{~mW}$ power at the fiber tip.

In vitro patch-clamp recordings. Four to 6 weeks after virus microinjection, mice were anesthetized with isoflurane and transcardially perfused with ACSF for slice preparation. Horizontal $350 \mu \mathrm{m}$ slices were obtained in bubbled ice-cold $95 \% \mathrm{O}_{2} / 5 \% \mathrm{CO}_{2}$ ACSF containing the following (in $\mathrm{mm}$ ): 110 choline chloride, 20 glucose, $2.5 \mathrm{KCl}, 0.5 \mathrm{CaCl}_{2}, 7 \mathrm{MgCl}_{2}, 1.3$ $\mathrm{NaH}_{2} \mathrm{PO}_{4}, 25 \mathrm{NaHCO}_{3}, 1.3 \mathrm{Na}$-ascorbate, and $0.6 \mathrm{Na}$-pyruvate. Slices were then incubated in ACSF containing the following (in mM): 10 glucose, $125 \mathrm{NaCl}, 2.5 \mathrm{KCl}, 2 \mathrm{CaCl}_{2}, 1.3 \mathrm{MgCl}_{2}, 1.3 \mathrm{NaH}_{2} \mathrm{PO}_{4}, 25 \mathrm{NaHCO}_{3}$, $1.3 \mathrm{Na}$-ascorbate, and $0.6 \mathrm{Na}$-pyruvate, at $34^{\circ} \mathrm{C}$ for at least $1 \mathrm{~h}$, and then kept at room temperature. Slices were transferred to a recording chamber that was continuously perfused with oxygenated ACSF $(4-6 \mathrm{ml} / \mathrm{min})$. Neurons were viewed under upright microscopy (Olympus X51W) and recorded with Axonpatch-700B amplifier (Molecular Devices). For most whole-cell recordings, the patch electrodes (6-8 $\mathrm{M} \Omega$ resistance) were filled with pipette solution containing (in $\mathrm{mM}$ ) $70 \mathrm{~K}$-gluconate, $70 \mathrm{KCl}, 2$ $\mathrm{NaCl}, 2 \mathrm{MgCl}_{2}$, 10 HEPES, 1 EGTA, 2 MgATP, $0.3 \mathrm{NaATP}$. In optogenetic experiments, blue collimated light-emitting diode with $465 \mathrm{~nm}$ peak wavelength (LD-1, Plexon) was used for photostimulation of channelrhodopsin (ChR2)-expressing ACC neurons. To evoke action poten- 
tials in the ACC neurons, we illuminated slices with blue light pulses of a $5 \mathrm{~ms}$ duration and $20 \mathrm{~Hz}$. In chemogenetic experiments, CNO was dissolved to the final concentration $(5 \mu \mathrm{M})$ in ACSF and delivered through the perfusion system.

Immunofluorescence. After behavior testing, mice were anesthetized with isoflurane and perfused transcardially with $200 \mathrm{ml} 0.05 \mathrm{M}$ sodium phosphate, $\mathrm{pH} 7.4$, containing $0.9 \% \mathrm{NaCl}$, followed by $300 \mathrm{ml} 4 \%$ paraformaldehyde in $0.05 \mathrm{M}$ sodium phosphate, $\mathrm{pH} 7.4$, containing $0.9 \%$ $\mathrm{NaCl}$. Brains were removed and fixed overnight in the same solution. Serial ACC and hippocampal coronal sections $(40 \mu \mathrm{m})$ were made on a vibratome (Leica) in a bath of PBS. For immunocytochemistry, slices were permeabilized with $0.1 \%$ Triton X-100, blocked, and incubated overnight with $0.1 \%$ Triton X-100/10\% fetal bovine serum in PBS containing rabbit anti-c-fos antibody (1:500; Synaptic Systems, 226003; RRID:AB_2231974), rabbit anti-HA antibody (1:400; Cell Signaling Technology, 3724; RRID:AB_1549585). Subsequently, the sections were incubated with secondary antibodies goat anti-rabbit Cy3 (1:200; Jackson ImmunoResearch, 111-165-003; RRID:AB_2338000). Finally, cultures were counterstained with Hoechst (1:200; Sigma-Aldrich, B2883) to label the nuclei. Images were acquired using a confocal microscope (LSM 510, Zeiss) at identical settings at the highest intensity for each of the conditions. To determine the total number of $\mathrm{EGFP}^{+}$neurons per ACC when rAAV2/R-hSyn-EGFP-WPRE-pA was infused into the vHPC, the counts from sampled ACC sections (on every 4th section in a series of $40 \mu \mathrm{m}$ coronal sections) were averaged and the mean values were multiplied by the total number of ACC sections.

Experimental design and statistical analysis. The sample size was predetermined by analyzing pre-experimental data with PASS (power analysis and sample size) software. For animal studies, the number of animals for each group was predetermined according to numbers reported in published studies or our prior experiment and the accurate animal numbers are given in figure legends. Investigators were blind to treatment group when assessing the outcome. Statistical tests were performed with IBM SPSS Statistics v19 software. All data are expressed as mean \pm SEM. Comparisons among multiple groups were made with two-way ANOVA or three-way ANOVA followed by Bonferroni post hoc test. Comparisons between two groups were made with a two-tailed Student's $t$ test. Statistical significance was set at $p<0.05$.

\section{Results}

\section{The ACC and vHPC are implicated in contextual} fear generalization

To establish a behavioral paradigm to successfully make fear memory generalized at a remote time point, we placed mice in a T-Ctx for 3 min acclimation to record baseline freezing values $(7.9 \pm 0.8 \%)$, and performed contextual fear-conditioning training by delivering three foot shocks via a grid floor. Four hours later, the mice were randomly divided into four groups: recent recall (retrieval $24 \mathrm{~h}$ after training) in T-Ctx and N-Ctx and remote recall (retrieval $14 \mathrm{~d}$ after training) in T-Ctx and N-Ctx. Fear retrieval was performed 1 or $14 \mathrm{~d}$ after training. The fear retrieval consisted of a 3 min exposure in T-Ctx or N-Ctx. At $1.5 \mathrm{~h}$ after retrieval, we randomly selected subset of animals ( 5 animals from each group) to detect C-fos ${ }^{+}$cells in the ACC, dHPC, and vHPC using immunohistochemistry method (Fig. 1A). For recent recall, freezing level in N-Ctx was significantly lower than that in T-Ctx, showing a contextually precise fear memory at the recent time point, and for remote recall, however, there were similar freezing levels between T-Ctx and N-Ctx, indicating a substantial contextual fear generalization (Fig. 1B). In T-Ctx, the numbers of $\mathrm{C}$-fos ${ }^{+}$cells in the ACC, dHPC, or vHPC were similar between recent and remote recall groups (Fig. 1C,D). In $\mathrm{N}$-Ctx, the number of $\mathrm{C}$-fos ${ }^{+}$cells in remote recall group was significantly increased in the ACC and vHPC, and similar in the dHPC compared with recent recall group (Fig. $1 C, E$ ), suggesting activation of ACC and vHPC in the N-Ctx at the remote time point. Thus, the ACC and vHPC may be recruited or involved in memory generalization of contextual fear conditioning.

\section{Activity of the ACC and vHPC is necessary and sufficient for fear generalization}

To specifically evaluate the contribution of ACC, dHPC and vHPC neurons in contextual fear discrimination, we used designer receptors exclusively activated by designer drugs (DREADDs) to selectively silence or stimulate the activity of ACC, dHPC, or vHPC neurons. We used AAV9-expressing Gicoupled hM4Di (AAV ${ }_{9}$-hSyn-HA-hM4Di-IRES-mCitrine), a recombinant virus vector that silences neurotransmission in the presence of DREADD agonist CNO (Armbruster et al., 2007). In addition to hM4Di, this viral vector also expressed a fluorescent protein, mCitrine, independent of hemagglutinin (HA)-hM4Di in the infected neurons using the internal ribosome entry site (IRES). Unlike HA-hM4Di, a transmembrane protein labeling neuronal processes including long projections, mCitrine is a soluble protein and labels the somata and proximal processes of the infected cells. We infused the viral vector into the ACC, dHPC, or vHPC by microinjection. Four weeks later, we placed mice in T-Ctx for $3 \mathrm{~min}$ to record baseline freezing values (ACC: $10.6 \pm$ 0.72\%; dHPC: $7.4 \pm 0.64 \%$; vHPC: $7.4 \pm 0.43 \%$ ), and performed the contextual fear-conditioning training. Four hours later, the mice for recent or remote recall were randomly divided into 4 groups: $\mathrm{CNO} / \mathrm{T}-\mathrm{Ctx}$, saline/T-Ctx, CNO/N-Ctx, and saline/N-Ctx. At $24 \mathrm{~h}$ after training for recent recall animals or $14 \mathrm{~d}$ after training for remote recall animals, $\mathrm{CNO}$ or saline was intraperitoneally injected. One hour later, we performed fear retrieval (Fig. 2A).

Four weeks after viral microinjection (Fig. $2 B-D$ ), infection of ACC, dHPC, and vHPC neurons was revealed by somatic mCitrine expression (Fig. $2 E-G$ ). Silencing the activity of ACC or vHPC neurons by CNO 1 or $14 \mathrm{~d}$ after training did not change freezing levels in T-Ctx compared with saline control (Fig. $2 \mathrm{H}, I$ ). These data suggest that the activity of neurons in the ACC or vHPC is not involved in the expression of fear memory. For recent recall, mice treated by $\mathrm{CNO}$ or saline displayed significantly lower freezing in N-Ctx than that in T-Ctx (Fig. $2 \mathrm{H}, I$ ), indicating a contextually precise fear retrieval. For remote recall, mice treated by saline displayed similar freezing level in T-Ctx and $\mathrm{N}-\mathrm{Ctx}$, suggesting fear generalization, and more importantly, silencing the activity of ACC or vHPC neurons by CNO significantly reduced freezing level in N-Ctx (vs T-Ctx), showing inhibition of fear generalization (Fig. $2 \mathrm{H}, \mathrm{I}$ ). Thus, the activity of ACC or vHPC neurons is necessary for fear memory generalization. Silencing the activity of dHPC neurons by CNO 1 or $14 \mathrm{~d}$ after training significantly reduced freezing levels in T-Ctx but not in N-Ctx, compared with saline control (Fig. $2 J$ ), which suggest that dHPC activation is important for expression but not for generalization of fear memory.

To test whether the reduced remote freezing retrieval of mice in N-Ctx after silencing the activity of ACC or vHPC results from modification of sensorimotor function or from changes in motivational emotional processes, we assessed locomotor activity of mice in an open-field test. The infusion of viral vector into the ACC, dHPC, or vHPC and contextual fear-conditioning training was the same as that in Figure 2. After training, the mice were randomly divided into $\mathrm{CNO}$ and saline control groups. $\mathrm{CNO}$ or saline was intraperitoneally injected $14 \mathrm{~d}$ after training. One hour later, locomotor activity of mice was measured (Fig. $3 A$ ). As shown in Figure $3 B$, the hM4Di/CNO system inhibited the firing of neurons in the ACC. Silencing the activity of ACC, vHPC, or dHPC did not change locomotor activity (Fig. $3 C$ ). 
A
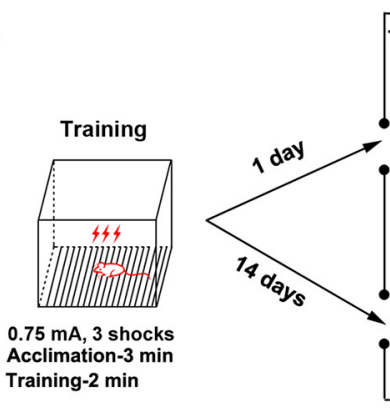

Training context

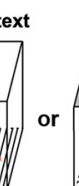

Novel context

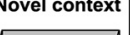

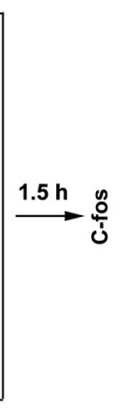

B

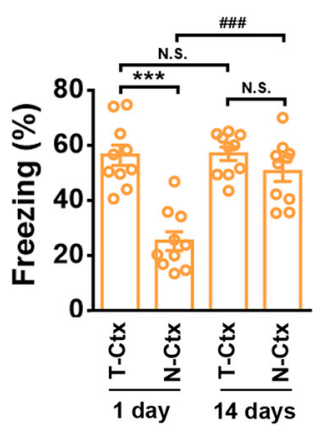

C

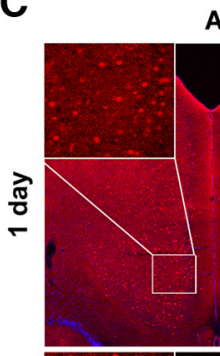

ACC

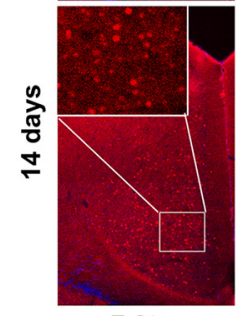

T-Ctx
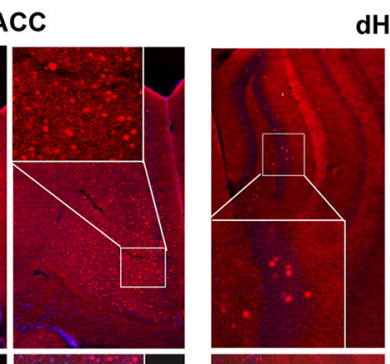

dHPC
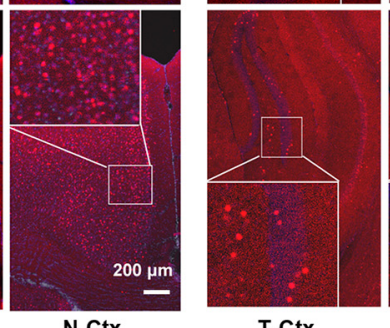

T-Ctx

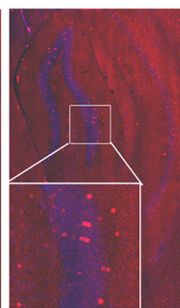

vHPC

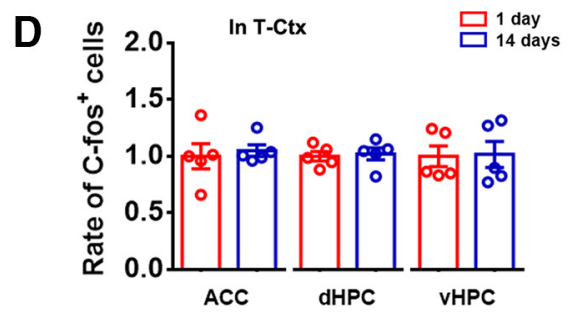

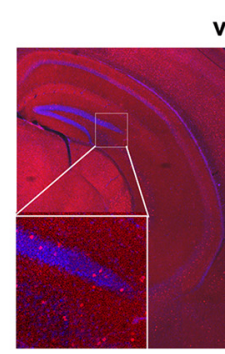

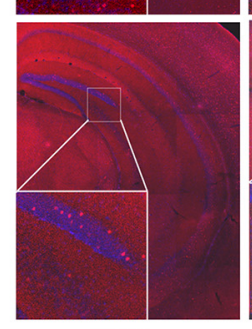

T-Ctx

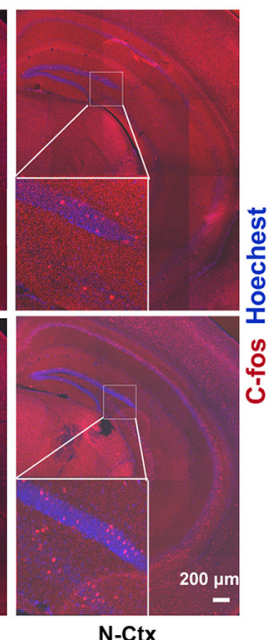

N-Ctx

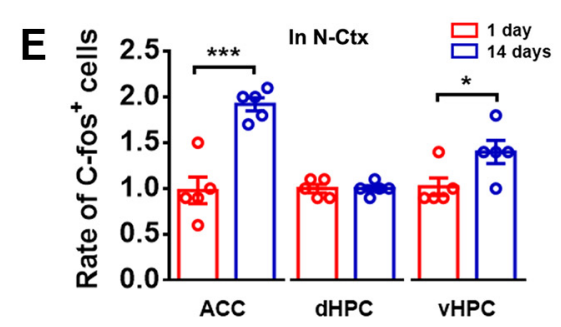

Figure 1. Implication of ACC and vHPC in fear generalization. $\boldsymbol{A}$, Design of the experiments for $\boldsymbol{B}-\boldsymbol{E}$. $\boldsymbol{B}$, Freezing levels of mice during recent and remote retrieval tests $(1$ and $14 \mathrm{~d}$ after training respectively) in T-Ctx or N-Ctx (two-way ANOVA followed by Bonferroni post hoc test; environment $F_{(1.36)}=32.134, p<0.001$; time $F_{(1.36)}=15.069, p<0.001$; interaction $F_{(1,36)}=13.969, p=0.001 ; n=10$ for each group; $\left.{ }^{* * *} p<0.001,{ }^{\# \# \#} p<0.001\right)$. C, Representative Immunofluorescence images of ACC, dHPC, and VHPC showing C-fos ${ }^{+}$cells and high-magnification images (top-left corner, ACC; lower-left corner, dHPC and vHPC) from selected areas. Red, C-fos; blue, Hoechst. $\boldsymbol{D}$, Number of c-fos ${ }^{+}$cells in ACC, dHPC, and vHPC of mice subjected to recent or remote fear retrieval in T-Ctx $\left(n=5 ;\right.$ ACC: $t_{(1 / 8)}=0.408, p=0.694 ; \mathrm{dHPC}: t_{(1 / 8)}=0.308, p=0.766 ; \mathrm{vHPC}: t_{(1 / 8)}=0.116, p=0.910$, two-tailed $t$ test). $E$, Number of c-fos ${ }^{+}$cells in ACC, dHPC, and vHPC of mice subjected to recent or remote fear retrieval in N-Ctx $\left(n=5 ; \mathrm{ACC}: t_{(1 / 8)}=5.718,{ }^{* * *} p<0.001 ; \mathrm{dHPC}: t_{(1 / 8)}=0.183, p=0.860\right.$; vHPC: $t_{(1 / 8)}=2.522,{ }^{*} p=0.036$, two-tailed $t$ test). N.S., no statistics significance, $p \geq 0.05$.

The motor cortex M2 is adjacent to ACC. To exclude the possibility that the contamination of $\mathrm{M} 2$ by virus vector may affect behavioral presentation, we infused $\mathrm{AAV}_{9}$-hSyn-HA-hM4Di-IRESmCitrine into the $\mathrm{M} 2$ by microinjection. Using similar a behavioral paradigm with Figure $2 A$, we detected freezing levels of mice $14 \mathrm{~d}$ after training in T-Ctx and in N-Ctx, and the activity of neurons infected by viral vector was silenced by $\mathrm{CNO}$ at $1 \mathrm{~h}$ before fear retrieval (Fig. 3D). Almost all the neurons infected by viral vector were restricted to the M2 (Fig. 3E). Silencing the activity of M2 neurons did not change freezing levels in T-Ctx or in N-Ctx, compared with saline control (Fig. 3F). Thus, the activity of M2 is not involved in the expression and generalization of fear memory.

To determine whether activity of ACC and vHPC neurons constitutes a sufficient condition for fear memory generalization, we used AAV9-expressing Gi-coupled hM3Dq $\left(\mathrm{AAV}_{9}\right.$-CaMKII $\alpha$ -
hM3Dq-mCherry), in which, CaMKII $\alpha$-driven hM3D(Gq) receptor with an mCherry reporter activates neurons in the presence of $\mathrm{CNO}$. We infused the viral vector into the ACC or vHPC by microinjection. Four weeks later, we placed mice in T-Ctx for $3 \mathrm{~min}$ to record baseline freezing values (ACC: $17.0 \pm$ $0.86 \%$; vHPC: $12.6 \pm 0.78 \%$ ), and performed the contextual fearconditioning training. At $24 \mathrm{~h}$ after training, $\mathrm{CNO}$ or saline was intraperitoneally injected. One hour later, we performed fear retrieval in T-Ctx or N-Ctx (Fig. 4A). Four weeks after viral injection, infection of ACC and vHPC was revealed by mCherry expression (Fig. $4 B$ ). As shown in Figure $4 C$, the $\mathrm{hM} 3 \mathrm{Dq} / \mathrm{CNO}$ system activated the firing of neurons in the ACC. In the saline-treated mice, freezing level in $\mathrm{N}$-Ctx was significantly lower than that in T-Ctx, showing a contextually precise fear memory. In the CNO-treated mice, however, retrieval in T-Ctx and in N-Ctx had similar freezing, and 
A

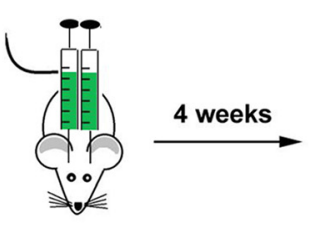

AAV9-hSyn-HA -hM4Di-IRES-mCitrine

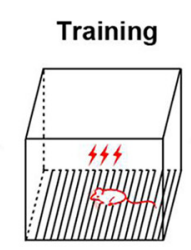

$0.75 \mathrm{~mA}, 3$ shocks Acclimation-3 $\mathrm{min}$ Training-2 $\mathrm{min}$

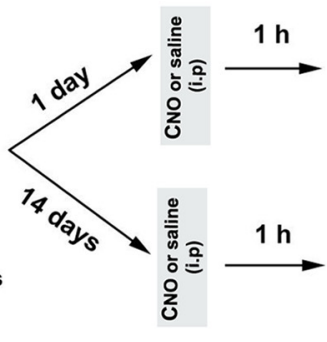

Training context

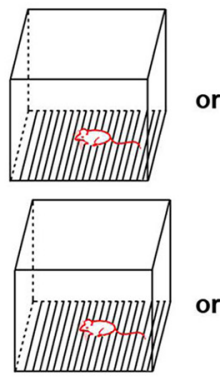

Retrieval-3 min
Novel context
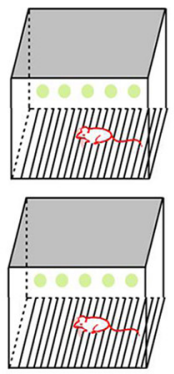
blue: hoechest

E ACC
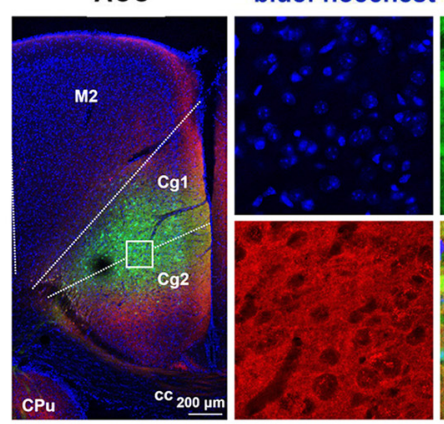

Red: HA, green: mcitrine

$\mathbf{F}$
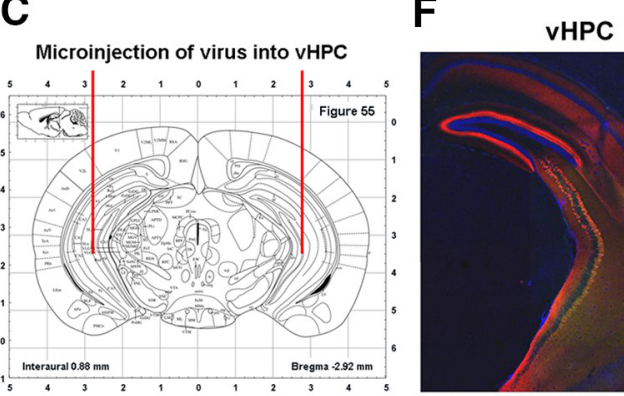
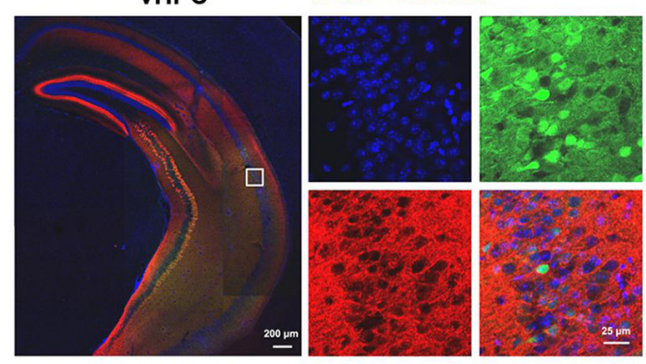

H
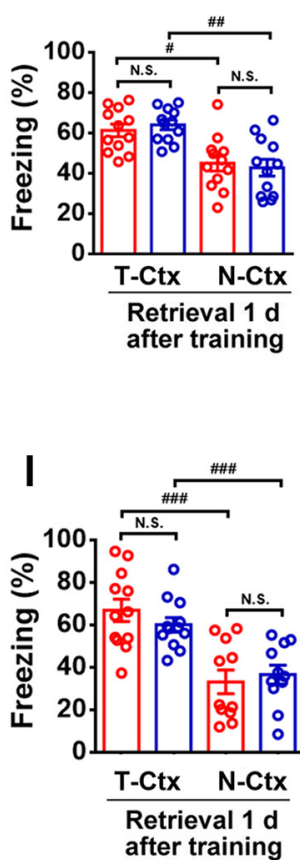

Retrieval $1 \mathrm{~d}$
after training
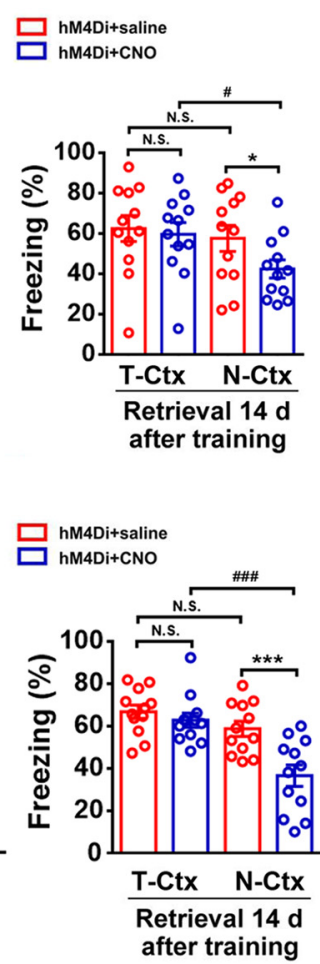

D

G $\quad$ dHPC

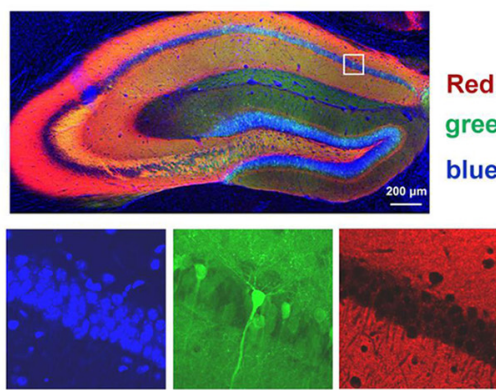

Red: HA

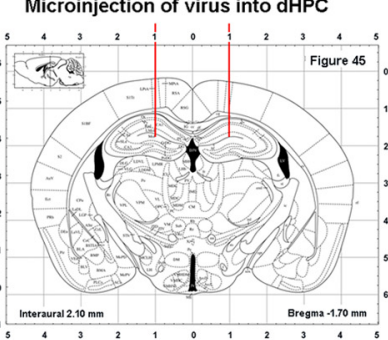

green: mcitrine blue: hoechest

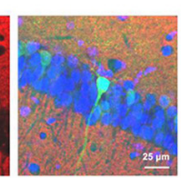

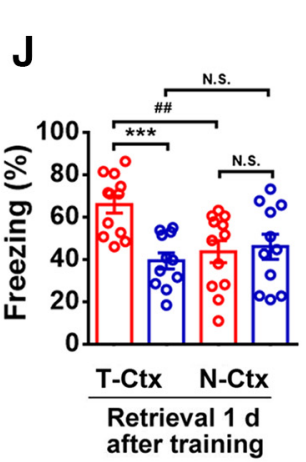

\section{hM4Di+salin}

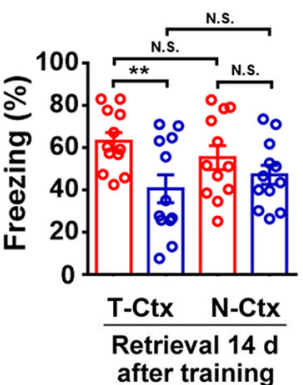

Figure 2. Activity of ACC or VHPC neurons is necessary for fear memory generalization. $\boldsymbol{A}$, Design of the experiments for $\boldsymbol{H}-\boldsymbol{J}$. $\boldsymbol{B}-\boldsymbol{D}, \mathrm{AAV}$ injection sites. $\boldsymbol{E}-\boldsymbol{G}$, Representative images ( $\boldsymbol{E}, \boldsymbol{F}$, left; $\boldsymbol{G}$, top) of the mice ACC, vHPC or dHPC infected by $\mathrm{AAV}_{9}$-hSyn-HA-hM4Di-IRES-m Citrine and high-magnification images $(\boldsymbol{E}, \boldsymbol{F}$, right; $\boldsymbol{G}$, bottom) from selected areas on the left $(\boldsymbol{E}, \boldsymbol{F})$ of top $(\boldsymbol{G})$ images. $\boldsymbol{H}-\boldsymbol{J}$, Freezing levels of mice retrieved $1 \mathrm{~d}$ (left) and $14 \mathrm{~d}$ (right) in T-Ctx or N-Ctx after training, in which the activity of neurons in the ACC, vHPC, or dHPC was silenced by CNO (i.p.) at $1 \mathrm{~h}$ before retrieval (three-way ANOVA followed by Bonferroni post hoc test. For ACC, treatment: $F_{(1,88)}=1.712, p=0.194$; environment: $F_{(1,88)}=19.276, p<0.001$; time: $F_{(1,88)}=0.433, p=0.512$; interaction: $F_{(1,88)}=0.299, p=0.586 ; n=12$ for each group; ${ }^{*} p<0.05,{ }^{\#} p<0.05$, ${ }^{\#} p<0.01$. For vHPC, treatment: $F_{(1,86)}=5.996, p=0.016$; environment: $F_{(1,86)}=57.007, p<0.001 ;$ time: $F_{(1,86)}=5.363, p=0.023$; interaction: $F_{(1,86)}=5.500, p=0.021, n=11$ for two groups in N-Ctx at the recent time point and $n=12$ for other groups; ${ }^{* * *} p<0.001$, \#\#\# $p<0.001$. For dHPC, treatment: $F_{(1,86)}=14.610, p<0.001$; environment: $F_{(1,86)}=1.351, p=0.248$; time: $F_{(1,86)}=0.563, p=0.455$; interaction: $F_{(1,86)}=1.047, p=0.309, n=11$ for two groups treated with CN0 at the recent time point and $n=12$ for other groups; ${ }^{* *} p<0.01,{ }^{* * *} p<0.001,{ }^{\# \#} p<0.01$. M2: secondary motor cortex; Cg1: cingulate cortex, area 1; Cg2: cingulate cortex, area 2; CPu: caudate putamen (striatum); cc: corpus callosum. N.S., no statistics significance, $p \geq 0.05$. 

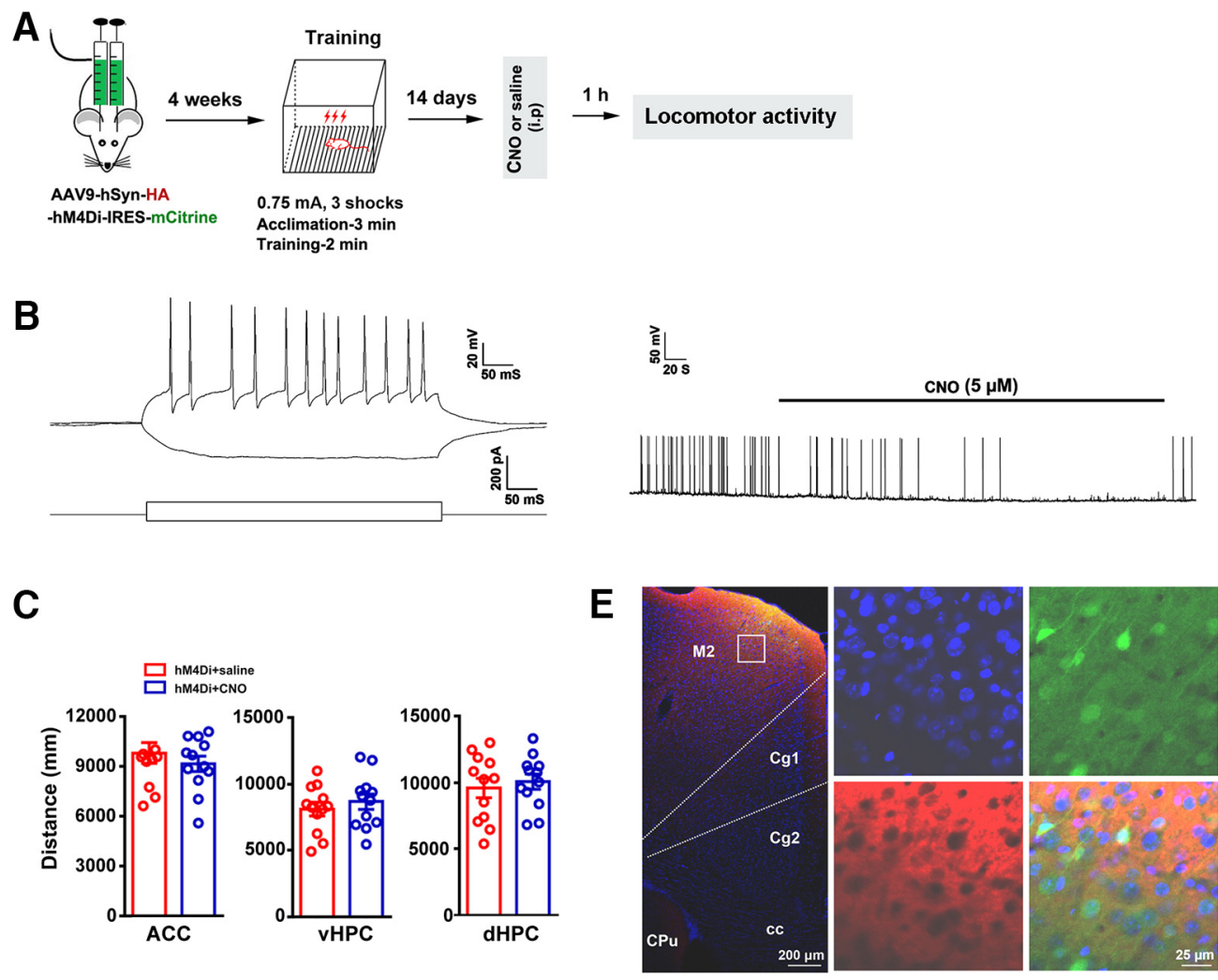

D
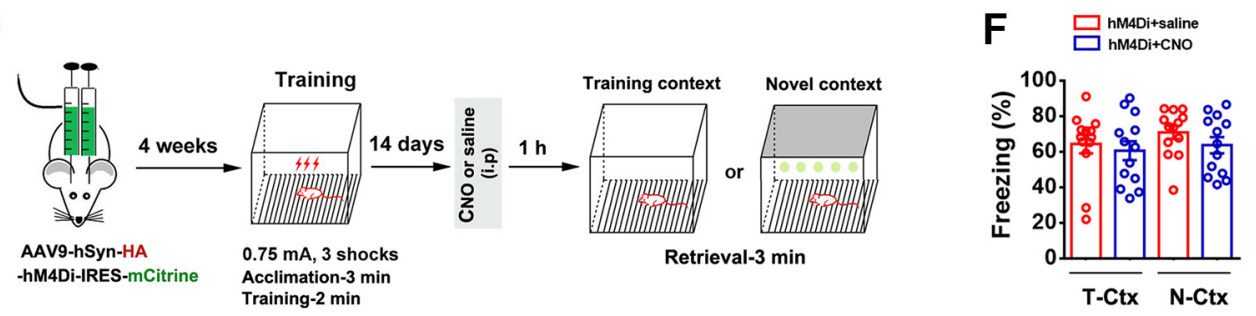

Figure 3. Silencing the activity of ACC, vHPC, or dHPC does not change locomotor activity and silencing the activity of M2 does not change freezing levels. $A$, Design of the experiments for $C$. $B$, The ability of the hM4D/CNO system to inhibit the firing of neurons in the ACC using in vitro whole-cell recordings. Left, Representative current-clamp traces of a neuron response to positive and negative $500 \mathrm{~ms}$ current injections ( -40 and $+120 \mathrm{pA}$, respectively). Right, In neurons expressing hM4-mCitrine, bath application of CNO completely abolished cell discharge of action potentials and this effect was reversed upon CNO washout. C, The locomotor activity in different groups $\left(n=12 ; \mathrm{ACC}: t_{(1 / 22)}=0.837, p=0.412 ; \mathrm{vHPC}: t_{(1 / 22)}=0.723, p=0.477 ; \mathrm{dHPC}: t_{(1 / 22)}=0.509, p=\right.$ 0.616 , two-tailed $t$ test). $\boldsymbol{D}$, Design of the experiments for $\boldsymbol{F}$. $\boldsymbol{E}$, Representative image of the mice M2 infected by recombinant virus AAV -hSyn-HA-hM4Di-IRES-mCitrine (left) and highmagnification images (right) from selected areas of the left image. $\boldsymbol{F}$, Freezing levels of mice retrieved $14 \mathrm{~d}$ (right) in $\mathrm{T}-\mathrm{Ctx}$ or $\mathrm{N}$-Ctx after training, in which the activity of neurons in the $\mathrm{M} 2$ were silenced by CNO (i.p.) at $1 \mathrm{~h}$ before retrieval (two-way ANOVA followed by Bonferroni posthoc test; treatment: $F_{(1,48)}=1.335, p=0.254$; environment: $F_{(1,48)}=1.014, p=0.319$; interaction: $F_{(1,48)}$ $=0.121, p=0.729 ; n=13$ for each group. M2: secondary motor cortex; Cg1: cingulate cortex, area 1; Cg2: cingulate cortex, area 2; CPu: caudate putamen (striatum); cc: corpus callosum.

moreover, in N-Ctx, CNO-treated mice exhibited significantly higher freezing than saline-treated mice (Fig. 4D,E).

Because there were mCherry expressions in both ACC and M2 (Fig. 4B), the hM3Dq/intraperitoneal CNO system-induced activation of neurons lacks selectivity of ACC. Moreover, it is reported that $\mathrm{CNO}$ does not cross the blood-brain barrier and DREADD-mediated behaviors after systemic CNO injections might be caused by clozapine (Gomez et al., 2017), and in addition, whether increasing the activity of ACC or vHPC neurons elevates basal freezing remains to be clarified. To address these, we performed photogenetic manipulation to activate ACC neurons selectively. We expressed ChR2 using viral-mediated transduction of neurons in the ACC and M2 and implanted optical fibers into the ACC (Fig. $4 F, G$ ). We trained mice in the contextual fear-conditioning paradigm and specifically stimulated the activity of ACC neurons by illumination with blue light (to activate ChR2; Fig. $4 F, H$ ). We detected freezing levels of mice $1 \mathrm{~d}$ before and after training in T-Ctx and in N-Ctx (Fig. 4F). We found that activating ACC neurons before training did not affect basal freezing levels of mice (Fig. 4I), whereas activating ACC neurons after training led to fear memory generalization (Fig. $4 J)$. Moreover, the basal freezing level (Fig. 4I) was similar to our previous study (Cai et al., 2018). Together, activation of the ACC and $v H P C$ is sufficient for fear generalization.

\section{Projections from the ACC to vHPC mediate fear memory generalization}

To test the projection relationship between the ACC and vHPC, a retrograde tracing adeno-associated virus (rAAV-hSyn-EGFP) was infused into the ACC or vHPC by microinjection. Six weeks later, infected neurons and surrounding nerve terminals in the ACC or vHPC were revealed by EGFP expression (Fig. $5 A-D$ ). When the virus was infused into the $\mathrm{ACC}$, no $\mathrm{EGFP}^{+}$neurons in the vHPC was observed (Fig. 5A, B). Contrastingly, when the virus was infused into 
A
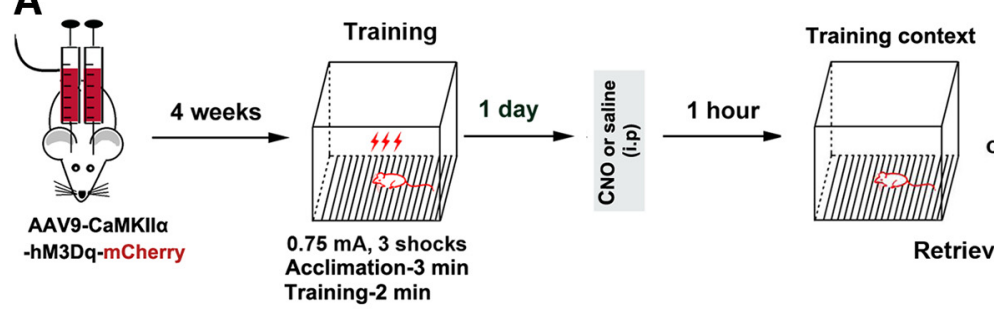

Training context

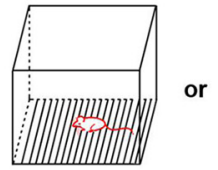

or

Retrieval-3 min
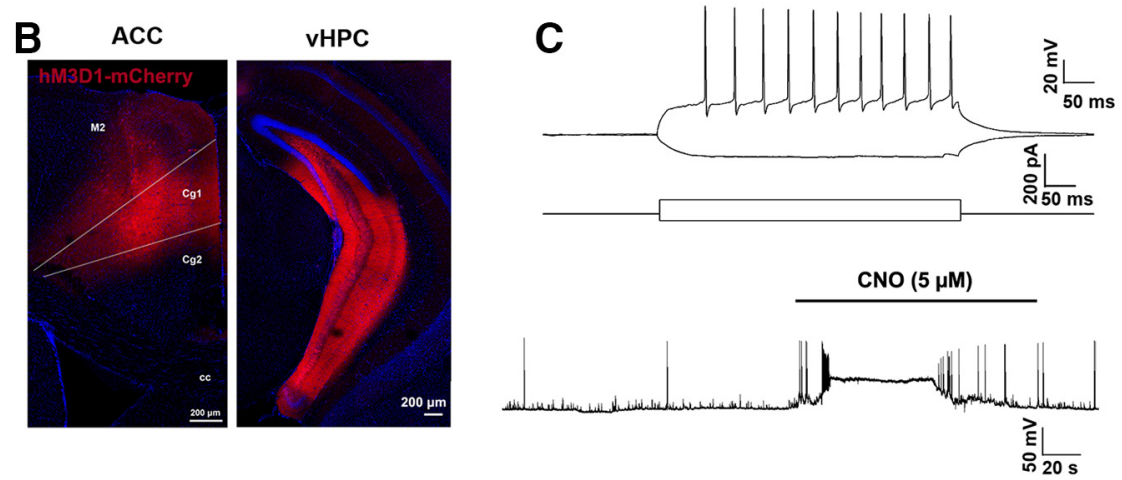

D $\square^{\text {nM3Dq+saline }}$
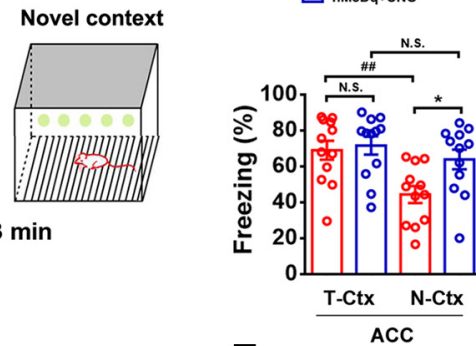

E

\section{$\square \mathrm{hM} 3 \mathrm{Dq}+\mathrm{saline}$}

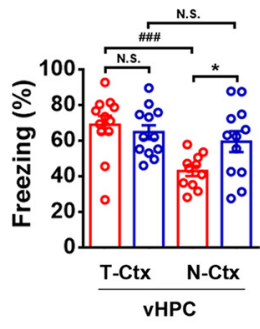

$\mathbf{F}$
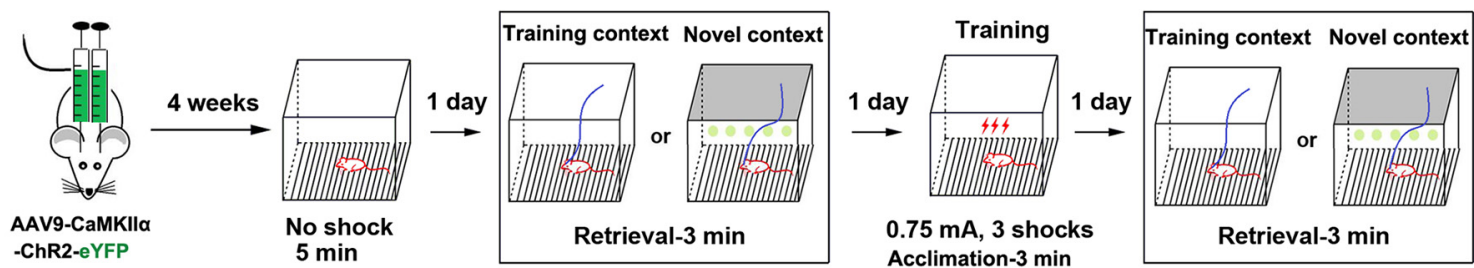

Acclimation-3 $\mathrm{min}$ Training-2 min

\section{G}

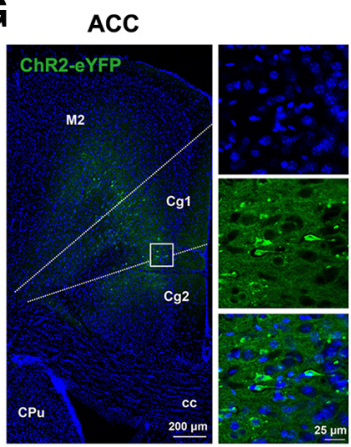

H

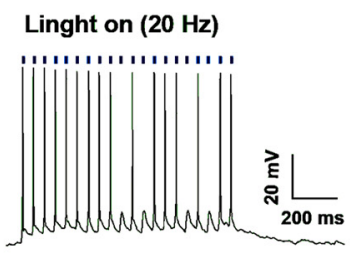

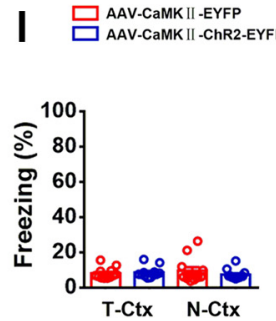

J吕 AAV-CaMKIII-EYFP

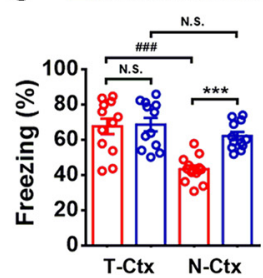

Figure 4. Activity of ACC and vHPC neurons is sufficient for fear memory generalization. $\boldsymbol{A}$, Design of the experiments for $\boldsymbol{D}$ and $\boldsymbol{E}$. $\boldsymbol{B}$, Representative images of the mice $A C C$ or vHPC infected by $\mathrm{AAV}_{9}$-CaMKIII $\alpha$-hM3Dq-mCherry. C, The ability of the hM3D/CNO system to activate the firing of neurons in the ACC using in vitro whole-cell recordings. Top, Representative current-clamp traces of a neuron response to positive and negative $500 \mathrm{~ms}$ current injections ( -40 and $+120 \mathrm{pA}$, respectively). Bottom, In neurons expressing hM3-mcherry, bath application of CNO elicited action potential firing and this effect was reversed upon CNO washout. D, E, Freezing levels of mice retrieved $1 \mathrm{~d}$ in T-Ctx or N-Ctx after training, in which ACC (D) or vHPC (E) neurons were activated by CN0 at $1 \mathrm{~h}$ before retrieval (two-way ANOVA followed by Bonferroni post hoc test; ACC, treatment: $F_{(1,44)}=4.697, p=0.036 ;$ environment: $F_{(1,44)}=9.958, p=0.003$; interaction: $F_{(1,44)}=2.710, p=0.107 ; n=12$ for each group; ${ }^{*} p<0.05,{ }^{\# \#} p<0.01 ; \operatorname{vHPC}$, treatment: $F_{(1,43)}=1.771, p=0.190 ;$ environment: $F_{(1,43)}$ $=11.392, p=0.002$; interaction: $F_{(1,43)}=4.960, p=0.031 ; n=11$ for the group treated with saline in the N-Ctx and $n=12$ for other groups; ${ }^{*} p<0.05$, $\left.{ }^{\# \# \#} p<0.001\right) . F$, Design of the experiments for $\boldsymbol{I}$ and $\boldsymbol{J}$. G, Images showing ChR2-eYFP-expressing ACC neurons (left) and high-magnification images from a selected area in the leftward image. $\boldsymbol{H}$, ChR2-mediated action potentials in ACC neuron were evoked by 5 ms pulses of light $(465 \mathrm{~nm})$ with $20 \mathrm{~Hz}$ in current-clamp mode. I, Freezing levels in T-Ctx or N-Ctx before training (two-way ANOVA followed by Bonferroni post hoc test; optogenetics: $F_{(1.44)}=0.462, p=0.500$; environment: $F_{(1.44)}=0.008, p=0.930$; interaction: $F_{(1.44)}=1.186, p=0.282 ; n=12$ for each group). $J$, Freezing levels in T-Ctx or N-Ctx after training (two-way ANOVA followed by Bonferroni post hoc test; optogenetics: $F_{(1,44)}=9.016, p=0.004 ;$ environment; $F_{(1,44)}=22.042, p=$ 0.000 ; interaction: $F_{(1.44)}=7.367, p=0.009 ; n=12$ for each group; ${ }^{* * *} p<0.001$, ${ }^{\# \#} p<0.001$. M2: secondary motor cortex; $\mathrm{Cg} 1$ : cingulate cortex, area 1; $\mathrm{Cg} 2$ : cingulate cortex, area 2; (Pu: caudate putamen (striatum); cc: corpus callosum. N.S., no statistics significance, $p \geq 0.05$.

the vHPC, $\mathrm{EGFP}^{+}$neurons in the ACC $(48 \pm 3.06 /$ per ACC; $n=8)$ were observed (Fig. $5 C, D$ ), suggesting unidirectional projections from the ACC to vHPC.

To further confirm this, we infused $\mathrm{AAV}_{9}$-hSyn-HA-hM4DiIRES-mCitrine into the vHPC to perform an anterograde tracing.
As a transmembrane protein, HA-hM4Di labeled neuronal processes including long projections to other brain areas. Four weeks after viral infusion, HA-labeled neurons and neural processes in the vHPC were revealed (Fig. 5E). As expected, no HA-labeled projection fiber was observed in the ACC (Fig. 
ACC
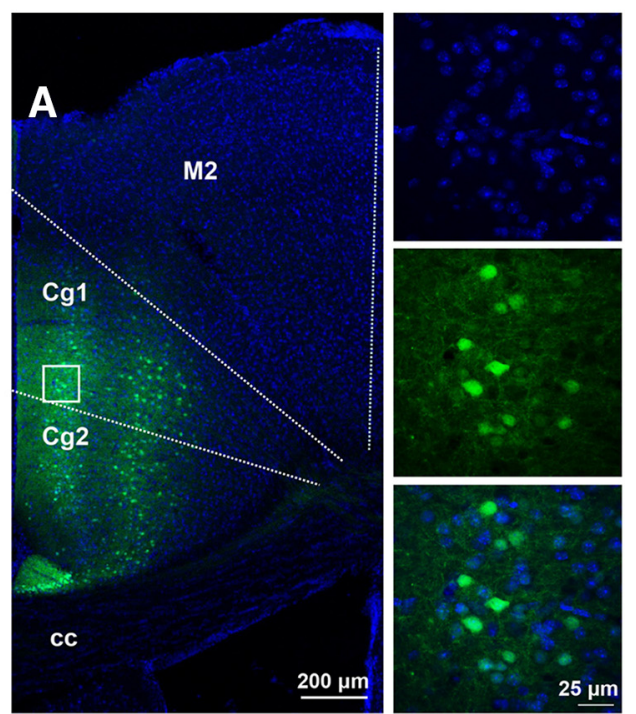

vHPC

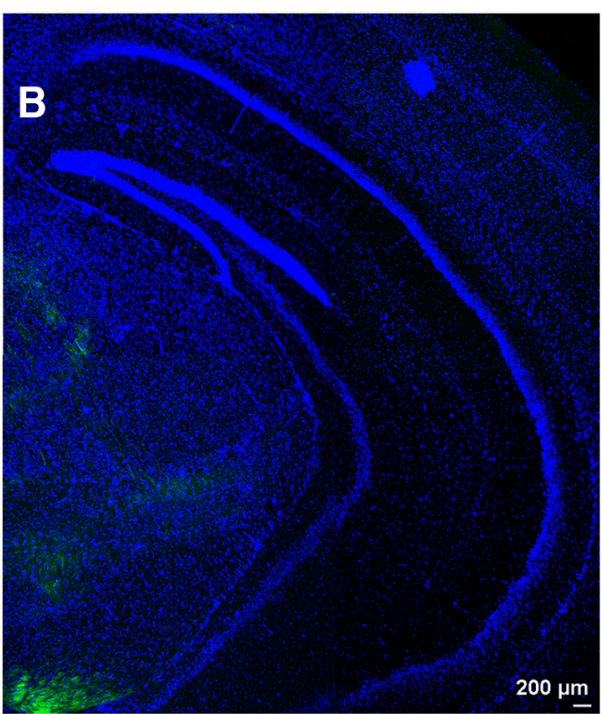

Green: rAAV-hsyn-EGFP, blue: hoechest

vHPC

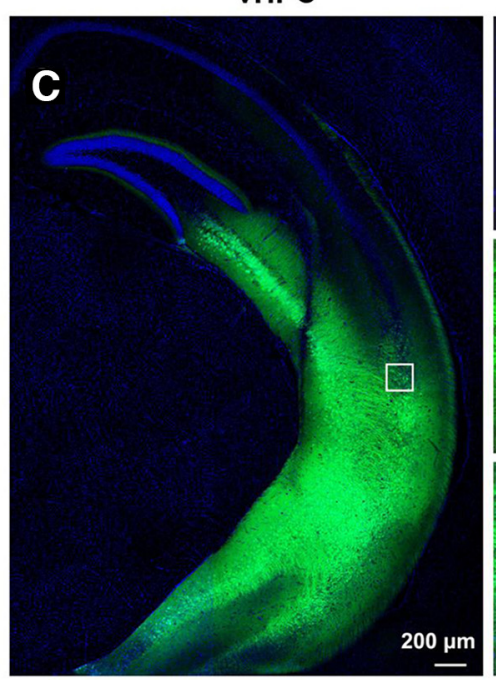

Green: rAAV-hsyn-EGFP, blue: hoechest

vHPC

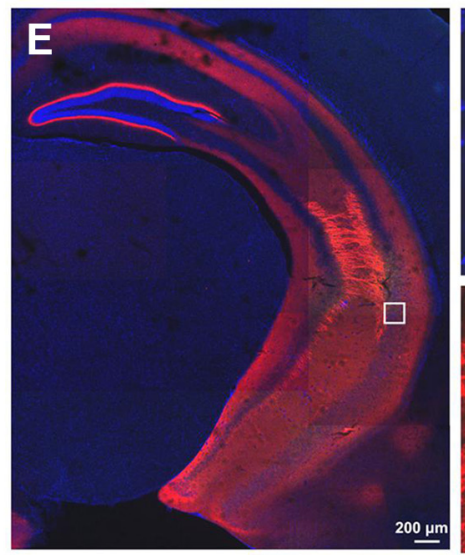

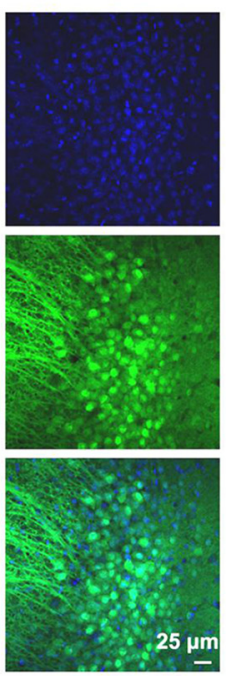

ACC

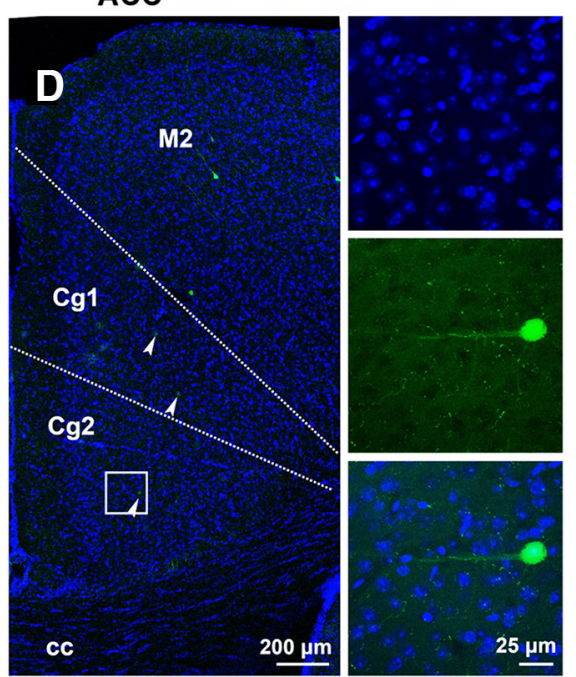

ACC
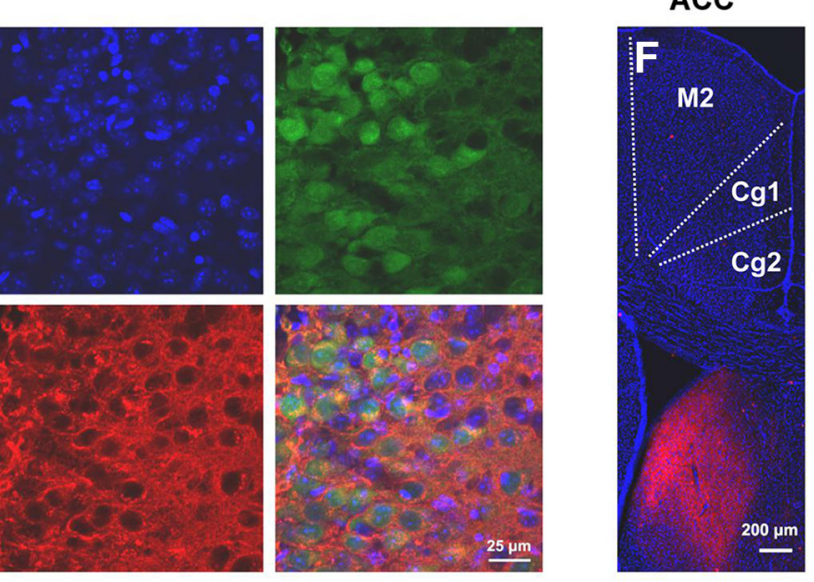

Red: HA, green: mCitrine, blue: hoechest

Figure 5. Projection pattern between the ACC and vHPC. $A$, Representative images of the ACC infected by rAAV-hSyn-EGFP (left) and high-magnification images (right) from selected areas in the left image. $\boldsymbol{B}$, Representative image of vHPC of mouse 6 weeks after receiving microinjection of rAAV-hSyn-EGFP into the ACC. C, Representative image of the vHPC infected by rAAV-hSyn-EGFP (left) and high-magnification images (right) from selected areas in the left image. $\boldsymbol{D}$, Representative image of the ACC (left) of mouse 6 weeks after receiving microinjection (Figure legend continues.) 


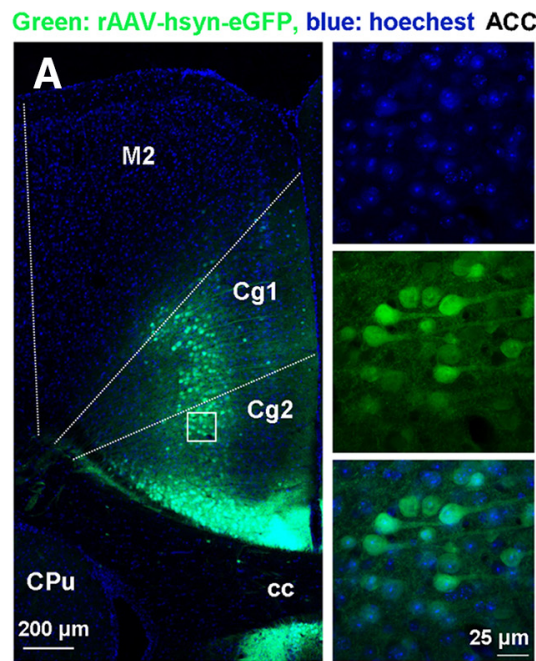

Green: rAAV-hsyn-eGFP, blue: hoechest dHPC

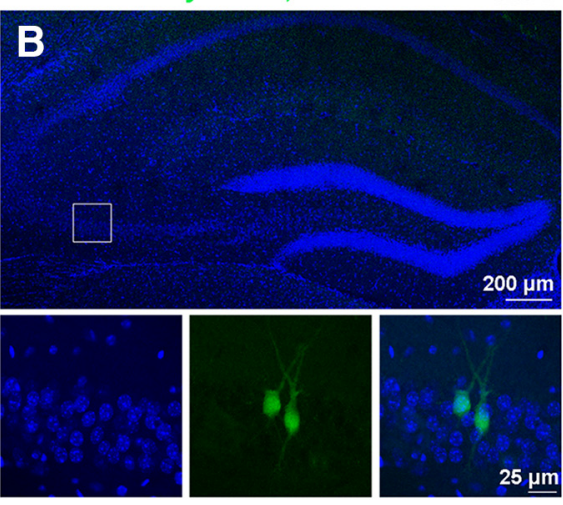

Green: rAAV-hsyn-eGFP, blue: hoechest dHPC Red: HA, green: mCitrine, blue: hoechest dHPC
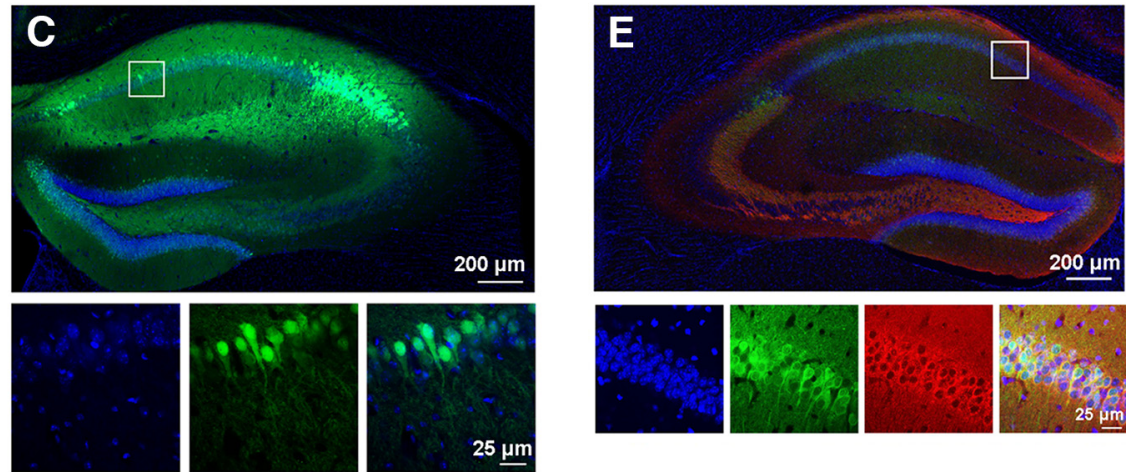

$5 \underline{\mu \mathrm{m}}$

Green: rAAV-hsyn-eGFP, blue: hoechest ACC
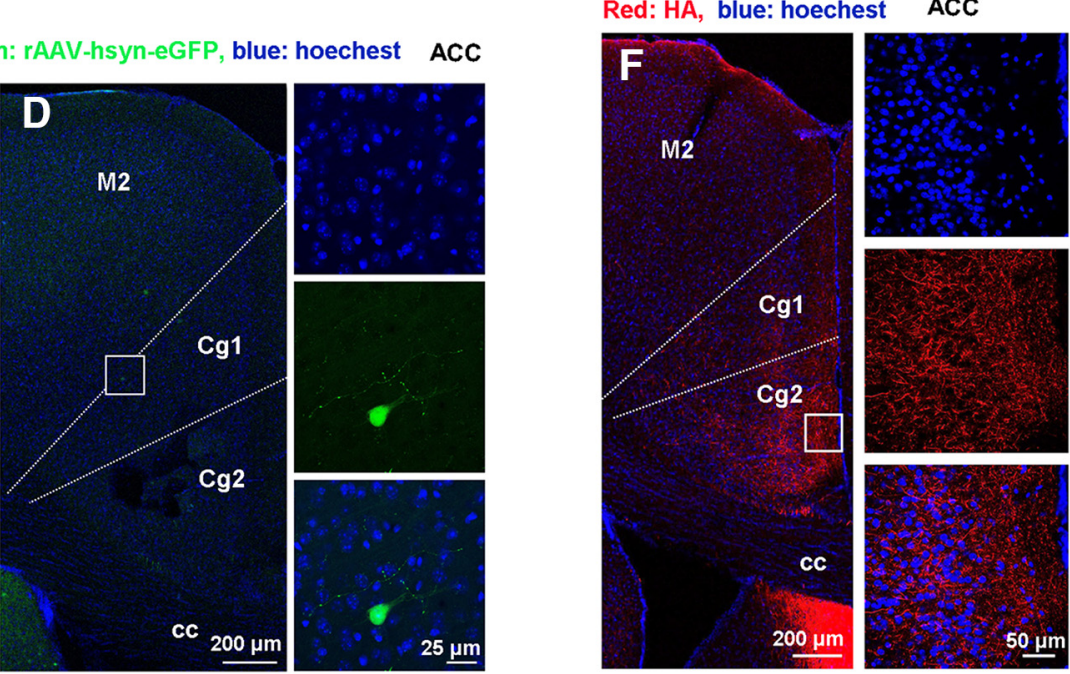

Figure 6. Projection pattern between the ACC and dHPC. $A$, Representative image of the ACC infected by rAAV-hSyn-EGFP (left) and high-magnification images (right) from selected areas in the left image. $\boldsymbol{B}$, Representative image of the dHPC (top) of mouse 6 weeks after receiving microinjection of rAAV-hSyn-EGFP into the ACC and high-magnification images (bottom) from selected areas in the top image. C, Representative image of the dHPC infected by rAAV-hSyn-EGFP (top) and high-magnification images (bottom) from selected areas in the top image. $\boldsymbol{D}$, Representative image of the ACC (left) of mouse 6 weeks after receiving microinjection of rAAV-hSyn-EGFP into the dHPC and high-magnification images (right) from selected areas in the left image. $E$, Representative image of the dHPC infected by AAV ${ }_{9}$-hSyn-HA-hM4Di-IRES-mCitrine (top) and high-magnification images (bottom) from selected areas in the top image. $F$, Representative image of ACC (left) of mouse 4 weeks after receiving microinjection of $\mathrm{AAV}_{9}$-hSyn-HA-hM4Di-IRES-mCitrine into the dHPC and high-magnification images (right) from selected areas in the left image. M2: secondary motor cortex; Cg1: cingulate cortex, area 1; Cg2: cingulate cortex, area 2; CPu: caudate putamen (striatum); cc: corpus callosum.

$5 F)$. Together, the ACC neurons project to the vHPC unidirectionally.

To determine the projection relationship between the ACC and dHPC, we infused rAAV-hSyn-EGFP into the ACC or dHPC by microinjection. Six weeks later, infected neurons and surrounding nerve terminals in the ACC or AHPC were revealed by EGFP expression (Fig. 6A-C). When rAAV-hSyn-EGFP was infused into the ACC (Fig. 6A), we found several EGFP ${ }^{+}$neurons in the dHPC (Fig. 6B), and when rAAV-hSyn-EGFP was infused into the $\mathrm{dHPC}$, many $\mathrm{EGFP}^{+}$neurons in the ACC were observed (Fig. 6C). Moreover, we infused $\mathrm{AAV}_{9}$-hSyn-HA-hM4Di-IRESmCitrine into the dHPC (Fig. 6D) and observed a large number of HA-labeled projection fibers in the ACC (Fig. 6E). Therefore, ACC and dHPC have neural projections between each other.

\section{$\leftarrow$}

(Figure legend continued.) of rAAV-hSyn-EGFP into the VHPC and high-magnification images (right) from selected areas in the left image. The white arrows indicate EGFP ${ }^{+}$. $\boldsymbol{E}$, Representative image of the vHPC infected by AAV $\mathrm{A}_{9}$ hSyn-HA-hM4Di-IRES-mCitrine (left) and highmagnification images (right) from selected areas in the left image. $\boldsymbol{F}$, Representative image of ACC of mouse 6 weeks after receiving microinjection of AAV $_{9}$-hSyn-HA-hM4Di-IRES-mCitrine into the VHPC. M2: secondary motor cortex; Cg1: cingulate cortex, area 1; Cg2: cingulate cortex, area 2; cc: corpus callosum.
To determine whether the neural projections from the ACC to vHPC mediate fear memory generalization, we infused $\mathrm{AAV}_{9}-$ hSyn-HA-hM4Di-IRES-mCitrine into the ACC. Four weeks later, we placed mice in T-Ctx for $3 \mathrm{~min}$ to record baseline freezing values $(5.8 \pm 0.69 \%)$, and performed the contextual fearconditioning training. $\mathrm{CNO}$ or saline was infused into the vHPC by microinjection $14 \mathrm{~d}$ after training. Thirty minutes later, we performed fear retrieval in T-Ctx or N-Ctx (Fig. 7A). Four weeks after viral infusion, Neurons expressing mCitrine and HAlabeled neural processes in the ACC and vHPC were revealed (Fig. $7 B, C$ ). In the saline-treated mice, there were similar freezing levels in $\mathrm{N}$-Ctx and in T-Ctx, suggesting a fear generalization. Interestingly, CNO-treated animals in $\mathrm{N}$-Ctx displayed significantly lower freezing than CNO-treated mice in T-Ctx and saline-treated animals in N-Ctx (Fig. 7D). Therefore, silencing the activity of projection fibers from the ACC to $\mathrm{vHPC}$ by CNO inhibited fear generalization at the remote time point.

\section{Discussion}

A number of studies have demonstrated that contextual fear memory becomes less context-specific over time, showing that animals increasingly generalize conditioned responding to N-Ctx (Biedenkapp and Rudy, 2007; Wiltgen and Silva, 2007; Wiltgen et 
A
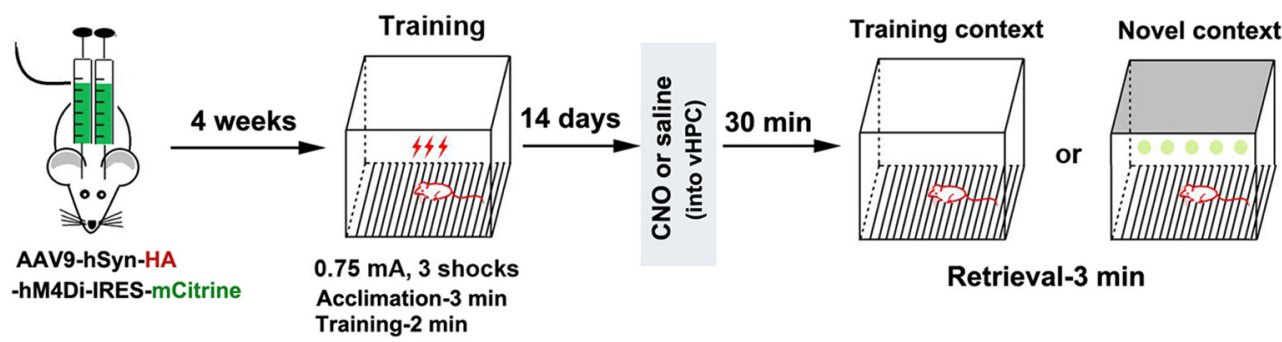

Retrieval-3 min
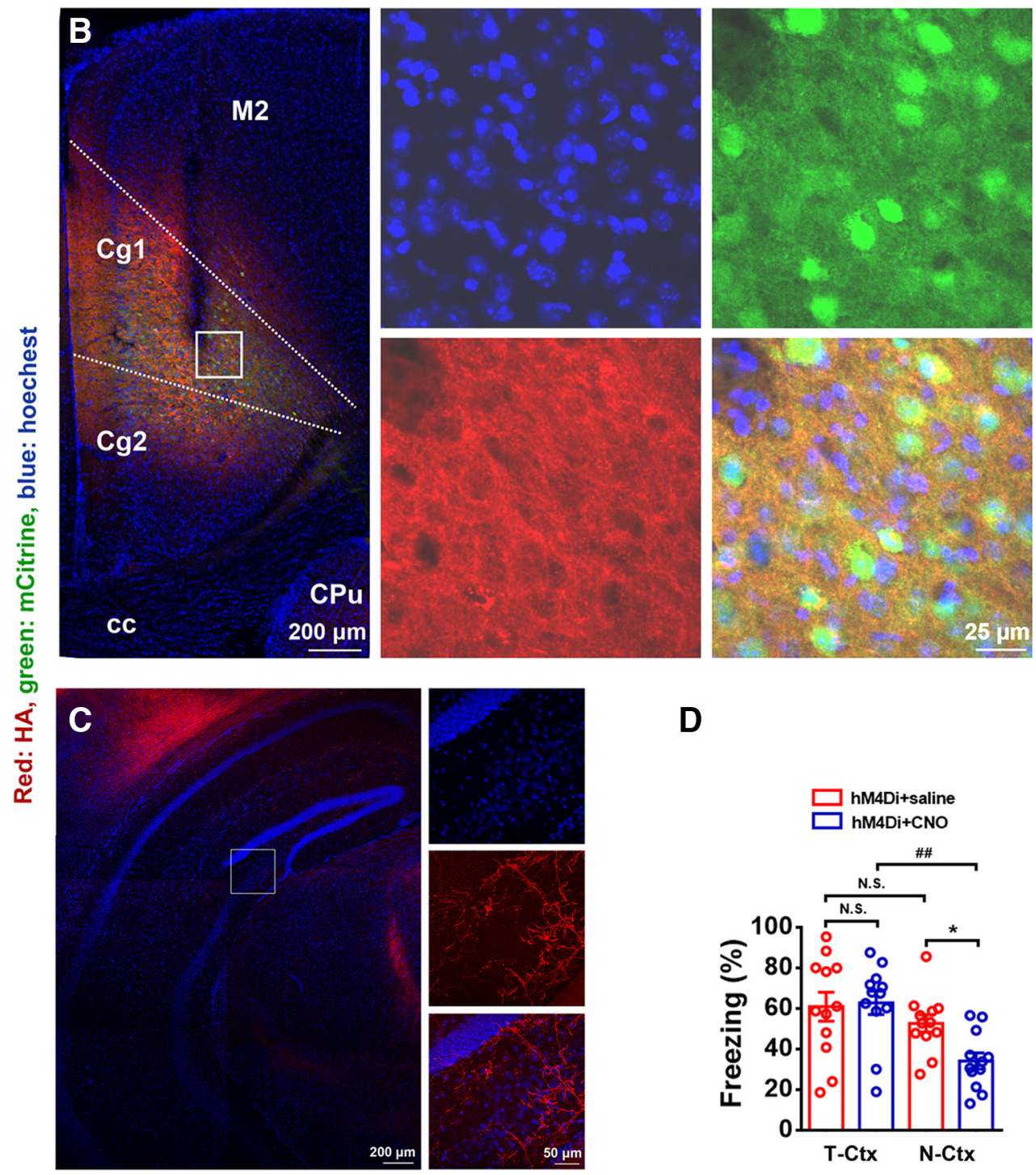

D

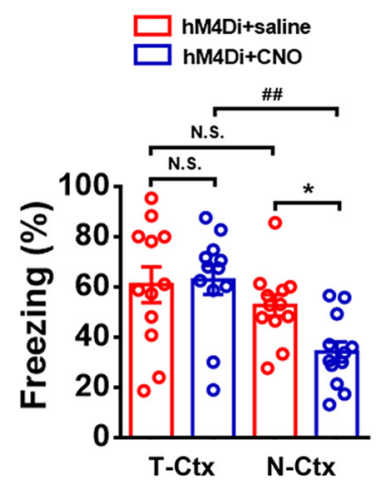

Figure 7. Neural projections from the ACC to vHPC mediate fear memory generalization. $\boldsymbol{A}$, Design of the experiments for $\boldsymbol{D}$. $\boldsymbol{B}$, Representative image of the $\mathrm{ACC}$ infected by AAV $-\mathrm{hS}$ Syn-HAhM4Di-IRES-mCitrine (left) and high-magnification images (right) from selected areas in the left image. C, Representative image of vHPC (left) of mouse 4 weeks after receiving microinjection of $\mathrm{AAV}_{9}$-hSyn-HA-hM4Di-IRES-mCitrine into the ACC and high-magnification images (right) from selected areas in the left image. D, Freezing levels of mice in T-Ctx or N-Ctx retrieved $14 \mathrm{~d}$ after training in which VHPC neurons receiving neural projections from the ACC were silenced by $\mathrm{CN} 0$ at 30 min before retrieval (two-way ANOVA followed by Bonferroni post hoc test; treatment: $F_{(1,44)}=2.340$, $p=0.133$; environment: $F_{(1,44)}=11.533, p=0.001$; interaction: $F_{(1,44)}=3.521, p=0.067 ; n=12$ for each group; $\left.{ }^{*} p<0.05,{ }^{\# \#} p<0.01\right)$. M2: secondary motor cortex; (g1: cingulate cortex, area 1; Cg2: cingulate cortex, area 2; CPu: caudate putamen (striatum); cc: corpus callosum. N.S., no statistics significance, $p \geq 0.05$.

al., 2010; Ruediger et al., 2011). Overgeneralization of contextual fear memory is associated with PTSD, but the circuit mechanisms underlying the generalization remain unclear. Here, our findings suggest that neural projections from the ACC to vHPC mediate contextual fear generalization, which is supported by four arguments: (1) significantly increased expression of C-fos occurred in the ACC and vHPC when contextual fear was retrieved in the
$\mathrm{N}$-Ctx at the remote time point, is consistent with the pattern of activity observed after remote memory activation in a previous report (Cullen et al., 2015); (2) silencing the activity of ACC or vHPC neurons reduced generalization to the same level seen in saline-treated animals at the remote time point, while stimulating the activity of ACC or VHPC neurons induced contextual fear generalization at the recent time point; (3) the ACC 
neurons project to the vHPC unidirectionally; and importantly, (4) silencing the activity of projection fibers from the ACC to vHPC inhibited contextual fear generalization at the remote time point.

The hippocampus is essential for the formation and retrieval of episodic and contextual memories in humans and animals. According to consolidation theory, the hippocampus plays a temporary role in the retrieval of the memories and they are being permanently stored in regions of the neocortex, including the ACC (Squire and Zola-Morgan, 1991; Kim and Fanselow, 1992; Alvarez et al., 1995). The dHPC is thought to normally underlie the formation of contextual fear memories (Lopez-Fernandez et al., 2007; Zelikowsky et al., 2013). We observed neural projections from the AHPC to the ACC (Fig. 6). Indeed, the dHPC is well positioned to inform the ACC regarding context through bottom-up pathways (Rajasethupathy et al., 2015). We showed here that there were bidirectional projections between the dHPC and ACC (Fig. 6). Based on the bidirectional projections, the ACC can access and mobilize engrams in the dHPC for top-down control of retrieval of recently encoded memory traces (Rajasethupathy et al., 2015). Silencing the activity of dHPC neurons significantly reduced retrievals of both recent and remote contextual freezing in the T-Ctx but not in N-Ctx (Fig. $2 J$ ). The dHPC is less activated during the retrieval of generalized context fear memories (Wiltgen et al., 2010; Fig. 1E). Based on these, we propose a model, in which, the memories regarding context are transferred from the dHPC to ACC through a bottom-up pathway, where they are being permanently stored. Precise context fear memories are retrieved in the dHPC through a top-down pathway from the ACC to dHPC (Fig. 8).

Up to now, most literature about contextual memory has focused on the dHPC, the potential role of the vHPC has been overlooked. By infusing sodium channel blocker lidocaine into the ACC and $\mathrm{vHPC}$, a recent study revealed that the activity of the ACC and vHPC is involved in the expression of a generalized fear memory (Cullen et al., 2015). Using chemogenetic and photogenetic manipulations in the behavioral paradigm, we demonstrated that activity of the ACC and vHPC is necessary and sufficient for contextual fear generalization (Figs. 2, 3). And more importantly, an ACC to vHPC neural circuit we identified here mediated contextual fear generalization (Fig. 7).

Usually, typical or substantial generalization occurs for remote events (Wiltgen and Silva, 2007; Jasnow et al., 2012; Wiltgen and Tanaka, 2013). So, we established a behavioral paradigm to successfully make fear memory generalized at $14 \mathrm{~d}$ after training (Fig. $1 A)$. Of course, memories are never completely precise but always partially generalized (Xu and Südhof, 2013). However, we are unable to prove whether slight generalization may occur at $1 \mathrm{~d}$ after training, because there is no reference. Moreover, there were different physical stimuli between T-Ctx and N-Ctx in our study, changes in freezing in N-Ctx may be caused by the stimulus features of that context. However, we found that the basal freezing levels were similar in the T-Ctx and N-Ctx (Fig. 4I), and that re- trieved freezing in T-Ctx and in N-Ctx $14 \mathrm{~d}$ after fear conditioning was similar, although freezing level in N-Ctx was significantly lower than that in T-Ctx $1 \mathrm{~d}$ after fear training (Fig. 1B). Thus, freezing modifications in N-Ctx is not caused by the stimulus features of that context.

How does contextual fear memory become generalized over time? A view is that hippocampus- and context-dependent original memory becomes transformed with time to one that is more schematic, which can explain contextual memories generalization, because in contextual fear conditioning, both contextually specific and nonspecific, the schematic information can be used to recall the fear response (Winocur et al., 2007). Thus, the retrieval of schematic information at remote time points is critical for fear generalization. The ACC is engaged early during the memory encoding process (Lesburguères et al., 2011; Tse et al., 2011; Bero et al., 2014) and has consistently been associated with the retrieval of contextual fear memory at remote time points (Frankland et al., 2004). Moreover, the ACC is implicated in memory generalization in humans (Maquet et al., 1996) and animals (Xie et al., 2013; Cullen et al., 2015). Based on that the ACC to vHPC circuit exists and silencing the activity of projection fibers from the ACC to vHPC inhibited contextual fear generalization at a remote time point (Figs. 5, 7 ), we suggest that, at remote time points, the vHPC can abstract gradually schematic elements of the fear memories stored in the ACC in N-Ctx for generalization through the ACC to vHPC circuit (Fig. 8).

Neural circuits contributing to contextual fear generalization could be complicated. In addition to the ACC to vHPC circuit we identified, a neural circuit composed of the mPFC, the thalamic $\mathrm{NR}$, and the hippocampus has been demonstrated to mediate memory generalization (Xu and Südhof, 2013). It is known that there are direct projections from vHPC to MPFC (Ye et al., 2017; Marek et al., 2018); thus, there may be a close relationship between the ACC to vHPC circuit and the mPFC-NR-hippocampus circuit, which needs to be investigated in the future. 


\section{References}

Allsop SA, Wichmann R, Mills F, Burgos-Robles A, Chang CJ, Felix-Ortiz AC, Vienne A, Beyeler A, Izadmehr EM, Glober G, Cum MI, Stergiadou J, Anandalingam KK, Farris K, Namburi P, Leppla CA, Weddington JC, Nieh EH, Smith AC, Ba D, et al. (2018) Corticoamygdala transfer of socially derived information gates observational learning. Cell 173: 1329-1342.e18.

Alvares Lde O, Einarsson EÖ, Santana F, Crestani AP, Haubrich J, Cassini LF, Nader K, Quillfeldt JA (2012) Periodically reactivated context memory retains its precision and dependence on the hippocampus. Hippocampus 22:1092-1095.

Alvarez P, Zola-Morgan S, Squire LR (1995) Damage limited to the hippocampal region produces long-lasting memory impairment in monkeys. J Neurosci 15:3796-3807.

Antunes R, Moita MA (2010) Discriminative auditory fear learning requires both tuned and nontuned auditory pathways to the amygdala. J Neurosci 30:9782-9787.

Armbruster BN, Li X, Pausch MH, Herlitze S, Roth BL (2007) Evolving the lock to fit the key to create a family of $\mathrm{G}$ protein-coupled receptors potently activated by an inert ligand. Proc Natl Acad Sci U S A 104:5163-5168.

Ashwell R, Ito R (2014) Excitotoxic lesions of the infralimbic, but not prelimbic cortex facilitate reversal of appetitive discriminative context conditioning: the role of the infralimbic cortex in context generalization. Front Behav Neurosci 8:63.

Bero AW, Meng J, Cho S, Shen AH, Canter RG, Ericsson M, Tsai LH (2014) Early remodeling of the neocortex upon episodic memory encoding. Proc Natl Acad Sci U S A 111:11852-11857.

Biedenkapp JC, Rudy JW (2007) Context preexposure prevents forgetting of a contextual fear memory: implication for regional changes in brain activation patterns associated with recent and remote memory tests. Learn Mem 14:200-203.

Bonne O, Grillon C, Vythilingam M, Neumeister A, Charney DS (2004) Adaptive and maladaptive psychobiological responses to severe psychological stress: implications for the discovery of novel pharmacotherapy. Neurosci Biobehav Rev 28:65-94.

Bontempi B, Laurent-Demir C, Destrade C, Jaffard R (1999) Timedependent reorganization of brain circuitry underlying long-term memory storage. Nature 400:671-675.

Cai CY, Chen C, Zhou Y, Han Z, Qin C, Cao B, Tao Y, Bian XL, Lin YH, Chang L, Wu HY, Luo CX, Zhu DY (2018) PSD-95-nNOS coupling regulates contextual fear extinction in the dorsal CA3. Sci Rep 8:12775.

Clelland CD, Choi M, Romberg C, Clemenson GD Jr, Fragniere A, Tyers P, Jessberger S, Saksida LM, Barker RA, Gage FH, Bussey TJ (2009) A functional role for adult hippocampal neurogenesis in spatial pattern separation. Science 325:210-213.

Cullen PK, Gilman TL, Winiecki P, Riccio DC, Jasnow AM (2015) Activity of the anterior cingulate cortex and ventral hippocampus underlie increases in contextual fear generalization. Neurobiol Learn Mem 124:19-27.

Dunsmoor JE, Otto AR, Phelps EA (2017) Stress promotes generalization of older but not recent threat memories. Proc Natl Acad Sci U S A 114: 9218-9223.

Einarsson EÖ, Pors J, Nader K (2015) Systems reconsolidation reveals a selective role for the anterior cingulate cortex in generalized contextual fear memory expression. Neuropsychopharmacology 40:480-487.

Fanselow MS, Dong HW (2010) Are the dorsal and ventral hippocampus functionally distinct structures? Neuron 65:7-19.

Frankland PW, Bontempi B, Talton LE, Kaczmarek L, Silva AJ (2004) The involvement of the anterior cingulate cortex in remote contextual fear memory. Science 304:881-883.

Gafford GM, Parsons RG, Helmstetter FJ (2013) Memory accuracy predicts hippocampal mTOR pathway activation following retrieval of contextual fear memory. Hippocampus 23:842-847.

Ghosh S, Chattarji S (2015) Neuronal encoding of the switch from specific to generalized fear. Nat Neurosci 18:112-120.

Gomez JL, Bonaventura J, Lesniak W, Mathews WB, Sysa-Shah P, Rodriguez LA, Ellis RJ, Richie CT, Harvey BK, Dannals RF, Pomper MG, Bonci A, Michaelides M (2017) Chemogenetics revealed: DREADD occupancy and activation via converted clozapine. Science 357:503-507.

Goshen I, Brodsky M, Prakash R, Wallace J, Gradinaru V, Ramakrishnan C, Deisseroth K (2011) Dynamics of retrieval strategies for remote memories. Cell 147:678-689.
Grosso A, Santoni G, Manassero E, Renna A, Sacchetti B (2018) A neuronal basis for fear discrimination in the lateral amygdala. Nat Commun 9:1214.

Guo N, Soden ME, Herber C, Kim MT, Besnard A, Lin P, Ma X, Cepko CL, Zweifel LS, Sahay A (2018) Dentate granule cell recruitment of feedforward inhibition governs engram maintenance and remote memory generalization. Nat Med 24:438-449.

Jasnow AM, Cullen PK, Riccio DC (2012) Remembering another aspect of forgetting. Front Psychol 3:175.

Kim JJ, Fanselow MS (1992) Modality-specific retrograde amnesia of fear. Science 256:675-677.

Lesburguères E, Gobbo OL, Alaux-Cantin S, Hambucken A, Trifilieff P, Bontempi B (2011) Early tagging of cortical networks is required for the formation of enduring associative memory. Science 331:924-928.

Lopez-Fernandez MA, Montaron MF, Varea E, Rougon G, Venero C, Abrous DN, Sandi C (2007) Upregulation of polysialylated neural cell adhesion molecule in the dorsal hippocampus after contextual fear conditioning is involved in long-term memory formation. J Neurosci 27:4552-4561.

Mahan AL, Ressler KJ (2012) Fear conditioning, synaptic plasticity and the amygdala: implications for posttraumatic stress disorder. Trends Neurosci 35:24-35.

Maquet P, Lejeune H, Pouthas V, Bonnet M, Casini L, Macar F, TimsitBerthier M, Vidal F, Ferrara A, Degueldre C, Quaglia L, Delfiore G, Luxen A, Woods R, Mazziotta JC, Comar D (1996) Brain activation induced by estimation of duration: a PET study. Neuroimage 3:119-126.

Marek R, Jin J, Goode TD, Giustino TF, Wang Q, Acca GM, Holehonnur R, Ploski JE, Fitzgerald PJ, Lynagh T, Lynch JW, Maren S, Sah P (2018) Hippocampus-driven feed-forward inhibition of the prefrontal cortex mediates relapse of extinguished fear. Nat Neurosci 21:384-392.

McCullough KM, Morrison FG, Ressler KJ (2016) Bridging the gap: towards a cell-type specific understanding of neural circuits underlying fear behaviors. Neurobiol Learn Mem 135:27-39.

Rajasethupathy P, Sankaran S, Marshel JH, Kim CK, Ferenczi E, Lee SY, Berndt A, Ramakrishnan C, Jaffe A, Lo M, Liston C, Deisseroth K (2015) Projections from neocortex mediate top-down control of memory retrieval. Nature 526:653-659.

Rosen JB, Schulkin J (1998) From normal fear to pathological anxiety. Psychol Rev 105:325-350.

Rozeske RR, Jercog D, Karalis N, Chaudun F, Khoder S, Girard D, Winke N, Herry C (2018) Prefrontal-periaqueductal gray-projecting neurons mediate context fear discrimination. Neuron 97:898-910.e6.

Ruediger S, Vittori C, Bednarek E, Genoud C, Strata P, Sacchetti B, Caroni P (2011) Learning-related feedforward inhibitory connectivity growth required for memory precision. Nature 473:514-518.

Squire LR, Zola-Morgan S (1991) The medial temporal lobe memory system. Science 253:1380-1386.

Tse D, Takeuchi T, Kakeyama M, Kajii Y, Okuno H, Tohyama C, Bito H, Morris RG (2011) Schema-dependent gene activation and memory encoding in neocortex. Science 333:891-895.

Wang DV, Ikemoto S (2016) Coordinated interaction between hippocampal sharp-wave ripples and anterior cingulate unit activity. J Neurosci 36:10663-10672.

Wiltgen BJ, Silva AJ (2007) Memory for context becomes less specific with time. Learn Mem 14:313-317.

Wiltgen BJ, Tanaka KZ (2013) Systems consolidation and the content of memory. Neurobiol Learn Mem 106:365-371.

Wiltgen BJ, Zhou M, Cai Y, Balaji J, Karlsson MG, Parivash SN, Li W, Silva AJ (2010) The hippocampus plays a selective role in the retrieval of detailed contextual memories. Curr Biol 20:1336-1344.

Winocur G, Moscovitch M, Sekeres M (2007) Memory consolidation or transformation: context manipulation and hippocampal representations of memory. Nat Neurosci 10:555-557.

Xie K, Kuang H, Tsien JZ (2013) Mild blast events alter anxiety, memory, and neural activity patterns in the anterior cingulate cortex. PLoS One 8:e64907.

Xu W, Südhof TC (2013) A neural circuit for memory specificity and generalization. Science 339:1290-1295.

Ye X, Kapeller-Libermann D, Travaglia A, Inda MC, Alberini CM (2017) Direct dorsal hippocampal-prelimbic cortex connections strengthen fear memories. Nat Neurosci 20:52-61.

Zelikowsky M, Bissiere S, Hast TA, Bennett RZ, Abdipranoto A, Vissel B, Fanselow MS (2013) Prefrontal microcircuit underlies contextual learning after hippocampal loss. Proc Natl Acad Sci U S A 110:9938-9943. 